FZR-37

April 1994

Preprint

S. Frauendorf and V.V. Pashkevich

General Axial Shapes of Sodium Clusters

subm. to Physical Review B 
Forschungszentrum Rossendorf e.V.

Postfach $510119 \cdot$ D-01314 Dresden

Bundesrepublik Deutșchland

Telefon (0351) 5913261 


\title{
General Axial Shapes of Sodium Clusters
}

\author{
S. Frauendorf and V.V.Pashkevich * \\ Institut für Kern- und Hadronenphysik, \\ Forschungszentrum Rossendorf e.V., \\ PF 510119, 01314 Dresden, Germany \\ and The Niels Bohr Institute, \\ Blegdamsvej 17, DK-Copenhagen $\varnothing$, Denmark
}

April 18, 1994

\begin{abstract}
The shell correction method is applied to $\mathrm{Na}$ clusters to calculate their shapes and binding energies. The equlibrium shapes are calculated minimizing simultanously five deformation parameters. Strong deviations from spheroidal shape including reflection asymmetric shapes are found. The influence of cluster deformation on the splitting of the dipole resonance and the separation energies is studied and compared with the available experimental data.
\end{abstract}

\section{Introduction}

Shortly after the discovery of sharp peaks in the abundance distributions of alkali clusters (magic numbers and shell structure) $[1,2,3]$ it was realized that the detailed $N$-dependence of the abundances or the ionization potentials can be understood as a. consequence of non-spherical cluster shapes [3,4]. Direct evidence for cluster deformation came from the splitting of the photo-absorption resonance (plasmon) $[3,5,6,7,8,9,10]$. All these effects are considered to be due to the quantization of the motion of the $s$-valence electrons through the whole cluster in a self-consistent potential. First estimates of the magnitude of the spheroidal shape distortions have been obtained by approximating the self-consistent potential by the modified harmonic oscillator model $[4,11]$. More general Kohn-Sham calculations assuming a spheroidal jellium ion background also predicted deformations of the light $\mathrm{Na}$ clusters up to $N=20$ [12]. Triaxial ellipsoidal shapes have been investigated using both the oscillator model [5,6] and Kohn-Sham calculations [13]. The approach based on the oscillator model is simple enough to allow the study of a wide range of clusters, but the estimates of the energies are only qualitative. The Kohn-Sham calculations with deformed jellium, on the other hand, are restricted to light clusters because of the large numerical effort needed. So far, the studies of equilibrium deformations

-On leave from LTP JINR, 141980 Dubna, Russian Federation 
have been restricted to the ellipsoidal shapes ${ }^{1}$. The study of nuclear shapes strongly suggests deviations from the ellipsoidal deformations (c.f. e.g. [14]). In the present paper the so-called microscopic-macroscopic shell correction method (SCM) $[14,15]$ developed in nuclear physics for general single-particle potentials is adapted to alkali clusters. It is very efficient and accurate enough to calculate the shape of metallic clusters in a wide mass range exploring a rather large class of axial shapes, which describe to sufficiently good approximation the most general axial shapes a cluster can assume when treated as the quantal droplet. The SCM with approximate analytic calculations of the "smooth" quantities, possible for the ellipsiodally deformed square well and oscillator potentials, was considered in refs. $[11,16,17]$. The SCM in its general formulation has been introduced to cluster physics by the present authors [18] who studied the family of axial shapes combining a quadrupole and a hexadecapole deformation using a Woods-Saxon electron potential. The SCM method was used in ref. [20] to study spheroidal cluster shapes by means of a modified oscillator potential tuned to spherical Kohn-Sham calculations.

The paper is composed as follows. Section 2 describes the shell correction theory adapted to alkali clusters. After a discussion of the physical background (2.1), the choice of the liquid drop energy and shape parameterization (2.2) is described. Then the average electron potential (2.3) and some technicalities (2.4) are presented. Section 3 contains the results of calculations for the five-parameter family of shapes in the mass range of $N=10$ to 310 . The physics of deformation is discussed. In particular, relations to the nuclear deformations are considered. The implications of our results for experimental quantities such as separation energies, abundances and the splitting of the photo-absorption resonance are discussed in section 4 .

\section{Shell correction for alkali clusters}

\subsection{The physical background}

The physical concept of the SCM applied to alkali clusters is the following one: The cluster is considered as a quantal droplet. Its total energy is divided into a classical part and a shell correction. The first is the energy of the classical droplet of liquid alkali metal, comprising the contributions of both the electrons and the ions. For neutral clusters, to which we restrict ourselves in the present study, its deformation dependence is exclusivly determined by the surface tension. The parameters of the liquid drop energy (surface tension and binding energy per atom) are not calculated but taken to be the experimental values for bulk metal. The classical drop prefers to be spherical. Deviations from the spherical shape are a consequence of the quantized motion of the delocalized $s$-valence electrons. The shell correction is the contribution to the valence-electron energy, which arises from the quantization of the electron motion. It is calculated from the energies of the valence electrons in the mean potential, which is generated by the ions together with the "non-quantized"

\footnotetext{
${ }^{1}$ Although in the Kohn-Sham calculations the electron density can take any shape, the restriction to ellipsoidal jellium does not allow for a free relaxation of the cluster shape.
} 
valence electrons. The mechanism that determines the shape is similar to the JahnTeller effect. Shapes for which the level density is small at the Fermi surface are energetically preferable. The actual deformation results from the competition between these quantal shell forces and the classical surface tension. The SCM treats this competition in a quantitative way permitting binding energies and deformation parameters to be calculated.

To quantify this discussion, let us formulate the SCM as an approximation to a jellium Kohn-Sham calculation $[11,16,17,19]$. Here, the total energy is given by the expression

$$
E=t+\frac{1}{2} \int d \tau d \tau^{\prime} \frac{\rho(\vec{r}) \rho\left(\vec{r}^{\prime}\right)}{\left|\vec{r}-\vec{r}^{\prime}\right|}+\mathcal{F}_{X}[\rho]+\int d \tau \rho(\vec{r}) V_{I}(\vec{r})+E_{I},
$$

where $t, \rho$ and $\mathcal{F}_{X}$ are the kinetic energy, the charge density and the exchange correlation energy functional of the valence electrons, respectively, whereas $V_{I}$ and $E_{I}$ are the Coulomb potential and the Coulomb energy of the jellium background of the positive ions, respectively. The valence-electron density is now divided into the smooth part $\tilde{\rho}$ and a small shell correction $\delta \rho$

$$
\rho=\tilde{\rho}+\delta \rho .
$$

The precise definition of the density of the non-quantized valence electrons $\tilde{\rho}$ is given in refs. $[14,15]$. It may be viewed as resulting from a Thomas-Fermi like treatment of the functional (1) (as in ref. [19]). Neglecting terms of the order $O\left(\delta \rho^{2}\right)$ the energy becomes

$$
E=\tilde{E}+\delta E
$$

where the expression for $\tilde{E}$ coincides with functional (1) substituting $\tilde{\rho}$ for its argument $\rho$.

The Strutinsky energy theorem [15] states that up to errors of the order $O\left(\delta \rho^{2}\right)$ the shell correction $\delta E$ is the difference between the energy of $N$ electrons in the average potential $\tilde{V}$ and the "smooth" energy of $N$ non-quantized electrons in the same potential

$$
\delta E=\sum_{i \leq N} e_{i}-\int^{\bar{\lambda}} \operatorname{de} \tilde{g}(e) e
$$

where $e_{i}$ are the eigenvalues of the electron hamiltonian

$$
\begin{gathered}
h=\frac{\vec{p}^{2}}{2 m}+\tilde{V} \\
\tilde{V}(\vec{r})=\int d \tau^{\prime} \frac{\tilde{\rho}\left(\vec{r}^{\prime}\right)+\rho_{I}\left(\vec{r}^{\prime}\right)}{\left|\vec{r}-\vec{r}^{\prime}\right|}+\frac{\delta}{\delta \tilde{\rho}} \mathcal{F}[\tilde{\rho}(\vec{r})] .
\end{gathered}
$$

It is seen that $\tilde{V}$ is generated by the ionic background and the non-quantized valence electrons. 
The smooth level density $\tilde{g}(e)$ is calculated by folding the single-particle level density

$$
g^{s p}(e)=\sum_{i} \delta\left(e-e_{i}\right)
$$

with the smearing function $f\left(\left(e-e^{\prime}\right) / \gamma\right)$ (c.f. refs. $\left.[14,15]\right)$ such that the level bunching caused by the quantization is averaged out:

$$
\begin{aligned}
\tilde{g}(e) & =\gamma^{-1} \int_{-\infty}^{\infty} d e^{\prime} f\left(\left(e-e^{\prime}\right) / \gamma\right) g^{s p}\left(e^{\prime}\right) \\
& =\gamma^{-1} \sum_{i} f\left(\left(e-e_{i}\right) / \gamma\right),
\end{aligned}
$$

where the function $f$ can be expressed in terms of the Hermite polynomials $H_{k}$

$$
\begin{aligned}
f(x) & =\pi^{-\frac{1}{2}} \exp \left(-x^{2}\right) \sum_{k=0,2}^{2 m} H_{k}(0) H_{k}(x) /\left(2^{k} k !\right) \\
& =\pi^{-\frac{1}{2}} \exp \left(-x^{2}\right) \sum_{k=0,2}^{2 m} a_{k} H_{k}(x), \\
a_{0} & =1, a_{k}=-\frac{1}{2} a_{k-2} / k \text { for } k \geq 2 .
\end{aligned}
$$

We use the smearing function of the order $m=3$. The choice of $\gamma$ is discussed below in subsection 2.4 .

The total energy is determined by $\tilde{E}$ and $h$. The simplification of the SCM as compared with to the full Kohn - Sham problem stems from the possibility to parameterize these two quantities, which then can be very efficiently calculated. Note, that there is no double counting of interaction terms as, e.g., in the unrenormalized sum of electron energies. Both physical intuition and Kohn-Sham calculations suggest that the non-quantized cluster should have a deformation energy $\tilde{E}=E_{L D}$ of a drop of liquid metal and the valence electrons move in potential $\tilde{V}=U$ with a flat bottom and a thin surface region. The potential and the drop are supposed to have the same shape but slightly different dimensions. The details of our choice of this parameterization are discussed below. It is noted that the partition of the energy into a smooth part and a shell correction does not depend on the validity of the local density approximation of the Kohn-Sham functional. By adjusting the parameters of $E_{L D}$ (for example, the value of the surface tension) one may correct for some possible deficiencies of this approximation.

The physics put into the parameterization of $\tilde{E}$ and $\tilde{V}$, which is basically the one of the jellium model, sets the frame of applicability of the SCM for clusters. It does not take into account the tendency of the ions to arrange themselves into geometrical patterns at low temperature [21, 22]. Hence, the physics of quantal droplets, described by the SCM is particularly relevant above a certain temperature (about $400^{\circ} \mathrm{K}$, which is roughly the melting temperature of bulk $\mathrm{Na}$ ). Below it, the results set a scale, which the geometrical arrangement energies can be compared to. 
Since the "freezing" goes gradually [21] there is a whole temperature range below $400^{\circ} \mathrm{K}$, for which one expects that the clusters behave basically like quantal droplets. In fact, this may even be the case for small clusters at zero temperature. The arrangement of atoms into geometrical patterns is expected to be most important for the deformation energy of the non-quantized droplet, $E_{L D}$. The shell effects are not expected to be modified dramatically, since the pseudopotentials of the $\mathrm{Na}$ ions are very weak [23].

Calculating the shell correction $\delta E$, the temperature of the valence electrons is assumed to be zero. This sets another limitation to the results. The thermal fluctuations will smooth the shapes we calculate for zero temperature. The temperature of clusters in typical experiments is about $45 \mathrm{meV}\left(500^{\circ} \mathrm{K}\right)$ or less. The fluctuations corresponding to this temperature do not very much modify the shape of light clusters of mass below 100 . For heavier clusters the thermal averaging will decrease the deformations.

It is noted that the "Shell correction through the kinetic - energy term" used in ref. [19] is identical with the above discussed general formulation of the SCM, which has been first applied to alkali clusters by the present authors [18]. The equivalence is easily seen by comparing eq. (4) with eqs. (20-22) of ref. [19] and realizing that the second term in eq. $(4)$ is $\operatorname{tr}(h \tilde{\rho})[14,15]$. The difference between the two approaches is that we parametrize $\tilde{E}$ determining the parameters from the experimental bulk properties whereas ref. [19] calculate $\tilde{E}$ by means of the Extended Thomas Fermi theory from the energy density functional. The latter is known to have problems to reproduce the experimental surface tension of bulk sodium.

\subsection{The liquid drop energy and the shape parameteriza- tion}

It was shown in the previous subsection that the total binding energy of a metallic cluster may be divided into the smooth part, $E_{L D}$, describing the total energy of clusters whose electron motion is not quantized and the shell correction, $\delta E$, that accounts for the bunching in the electron spectrum caused by the quantal nature of the electron motion, i.e.,

$$
E=E_{L D}+\delta E .
$$

For neutral clusters we write $E_{L D}$ as the energy of a droplet consisting of $N$ atoms

$$
E_{L D}=-a N+4 \pi r_{S}^{2} N^{2 / 3} \sigma S\left(\alpha_{\mu}\right) / S_{0} .
$$

Here $r_{S}$ is the Wigner-Seitz radius as given by the cluster volume

$$
V=\frac{4 \pi}{3} R_{0}^{3}, \quad R_{0}=r_{S} N^{1 / 3} .
$$

We use $r_{S}=2.17 \AA$, the value corresponding to the density of bulk $\mathrm{Na}$ at $500{ }^{\circ} \mathrm{K}[24]$. The first term in eq. (10) is the volume energy that is independent of the cluster shape. For the separation energy of one atom from bulk $\mathrm{Na}$ at $500{ }^{\circ} \mathrm{K}$ we adopt $a=1.063 \mathrm{eV}$, which is the enthalpy of evaporation of liquid $\mathrm{Na}$ into a gas 
of monomers [25]. The second term is the surface energy. It depends on the cluster shape which is described by the set of deformation parameters $\alpha_{\mu}$ (see below). It is the product of the surface energy of a sphere, $S_{0}$, with radius $R_{0}$ and the ratio $S / S_{0}$ of the areas of the surface fixed by the set $\alpha_{\mu}$ and a sphere of the same volume. For the surface tension we take $\sigma=0.177 \mathrm{~J} / \mathrm{m}^{2}=0.0111 \mathrm{eV} / \AA^{2}$, the value for bulk $\mathrm{Na}$ at $500^{\circ} \mathrm{K}[24]$.

The shape of the cluster is assumed to be axially symmetric. It is described by a two-dimensional surface enclosing the volume $V$. We use the family of surfaces introduced in ref. [26], where one may look for details. It is based on the sequence of Cassinian ovaloids labelled by the deformation parameter $\alpha$. The values $\alpha<$ $0,0,0<\alpha<1,1$ and $1<\alpha$ correspond, respectively, to an oblate shape, a sphere, a prolate shape with some neck, the scission shape looking like $\infty$ and two fragments. To conveniently describe an arbitrary axial shape close to the above mentioned Cassinian ovaloids a special orthogonal coordinate system is introduced in which one of the families of the coordinate lines is the Cassinian ovals. The connection of the coordinates in this system, $(\mathcal{R}, x)$, which may be called Cassinian coordinates, to the cylindrical coordinates, $(r, z)$, is given by the following relations [26]

$$
\begin{aligned}
& \mathcal{R}=\left[\left(z^{2}+r^{2}\right)^{2}-2 \varsigma\left(z^{2}-r^{2}\right)+\varsigma^{2}\right]^{\frac{1}{4}}, \\
& x=\frac{\operatorname{sign}(z)}{\sqrt{ } 2}\left\{1+\frac{z^{2}-r^{2}-\varsigma}{\left[\left(z^{2}+r^{2}\right)^{2}-2 \varsigma\left(z^{2}-r^{2}\right)+\varsigma^{2}\right]^{\frac{1}{2}}}\right\}^{\frac{1}{2}},
\end{aligned}
$$

with the inverse transformation

$$
\begin{aligned}
& r=\frac{1}{\sqrt{ } 2}\left[\left(\mathcal{R}^{4}+2 \varsigma \mathcal{R}^{2}\left(2 x^{2}-1\right)+\varsigma^{2}\right)^{\frac{1}{2}}-\mathcal{R}^{2}\left(2 x^{2}-1\right)-\varsigma\right]^{\frac{1}{2}}, \\
& z=\frac{\operatorname{sign}(x)}{\sqrt{ } 2}\left[\left(\mathcal{R}^{4}+2 \varsigma \mathcal{R}^{2}\left(2 x^{2}-1\right)+\varsigma^{2}\right)^{\frac{1}{2}}+\mathcal{R}^{2}\left(2 x^{2}-1\right)+\varsigma\right]^{\frac{1}{2}},
\end{aligned}
$$

where $\varsigma$ is the squared distance from the focus of the Cassinian oval to the origin of the coordinates. In case of $\varsigma=0$ the Cassinian coordinates are the radius and cosine of the polar angle of the polar coordinate system. It is convenient to introduce instead of $\varsigma$ a dimensionless parameter $\varepsilon$,

$$
\varepsilon=\varsigma\left(c / R_{0}\right)^{2}
$$

The radius $R_{0}$ has been introduced earlier and $c\left(\alpha, \alpha_{\mu}\right)$ is chosen in such a way that the volume $V$ inside the surface $S$ is kept constant.

The deviation of the actual surface from the Cassinian ovaloid is represented by a sum of Legendre polynomials, $P_{\mu}$, weighted with the deformation parameters $\alpha_{\mu}$ as follows

$$
\mathcal{R}(x)=\frac{R_{0}}{c}\left(1+\sum_{\mu} \alpha_{\mu} P_{\mu}(x)\right)
$$

Thus the parameters $\left(\varepsilon, \alpha_{\mu}\right)$ completely characterize the cluster shape. To describe very deformed shapes that may be encountered in fission another parameter was 
introduced in ref. [26]. For a reflection-symmetric shape it is defined as follows

$$
\alpha=\left(z_{L}^{2}+z_{R}^{2}-2 \varrho\right) /\left(z_{L}^{2}+z_{R}^{2}+2 \varrho\right),
$$

where $z_{L(R)}$ is the minimum (maximum) value of $z$ on the surface and $\varrho=r_{\text {neck }}^{2}$ in case of single-connected body, $r_{n e c k}$ being the minimal radius of the cross-section of the surface in the plane perpendicular to the axis of symmetry. For two separated fragments, $\varrho$ is minus the square of half the distance between the tips of the fragments. The relation between $\alpha$ and $\varepsilon$ can be derived from eqs. (13) and (16)

$$
\begin{gathered}
\varepsilon=\frac{\alpha-1}{4}\left[\left(1+\sum_{\mu} \alpha_{\mu}\right)^{2}+\left(1+\sum_{\mu}(-1)^{\mu} \alpha_{\mu}\right)^{2}\right] \\
+\frac{\alpha+1}{2}\left[1+\sum_{\mu=1}(-1)^{\mu} \alpha_{2 \mu}(2 \mu-1) ! ! /\left(2^{\mu} \mu !\right)\right]^{2},
\end{gathered}
$$

which will be used also for asymmetric shapes. We will use the set of deformation parameters $\left(\alpha, \alpha_{\mu}\right)$.

For $|\alpha|<0.4$ the ovaloids approach spheroids and the shape parameterization is similar to standard multipole expansion relative to a sphere [27]. In this case $\alpha_{1}$ generates essentially a shift of the body as a whole along the symmetry axis and $\alpha_{2}$ is strongly correlated with $\alpha$. Thus for $|\alpha|<0.4$ we consider only $\mu \geq 3$. In what follows we will mainly be concerned with this case and, somewhat loosely speaking, we will refer to $\alpha, \alpha_{3}, \alpha_{4}, \ldots$ as to the quadrupole, octupole, hexadecapole, $\ldots$ deformations. For the family of reflection-symmetric shapes generated by $\alpha$ and $\alpha_{2 \mu}$ the ratio of the radius of the equator, $R_{\perp}=r_{\text {neck }}$, and the distance of the pole from the center, $R_{\|}=z_{R}$, is given by

$$
\frac{R_{\perp}}{R_{\|}}=\left[\frac{1-\alpha}{1+\alpha}\right]^{\frac{1}{2}} .
$$

This expression will also be used for asymmetric shapes, where $R_{\|}^{2}=\frac{1}{2}\left(z_{L}^{2}+z_{R}^{2}\right)$.

\subsection{The valence-electron hamiltonian}

In expression (5) for the single-valence-electron hamiltonian we set $m$ equal to the free electron mass since the effective mass of a valence electron in bulk $\mathrm{Na}$ approaches very closely to this value [23]. For a sufficiently large cluster, the potential $\tilde{V}=U$, which is generated jointly by the ions and the (non-quantized) electrons, is expected to be approximately constant inside and to disappear in a thin surface layer. We assume the thickness of the layer to be independent of the shape of the cluster, ${ }^{2}$ describing the surface profile by means of a Fermi function

$$
U(\vec{x})=U_{0} /[1+\exp (l(\vec{x}) / d)]
$$

\footnotetext{
${ }^{2}$ This approximation is consistent with the expression for the liquid drop energy (10) which may be viewed as an expansion into powers of $N^{-1 / 3}$. The next term would be the curvature energy that has its counterpart in a varying surface thickness.
} 
where $l(\vec{x})$ measures the distance of a point $\vec{x}$ from the the equipotential surface $U(l=0)=\frac{1}{2} U_{\circ}[14,28,29]$. The value of $l(\vec{x})$ is found numerically. The shape of this surface is the same as the one used to calculate $E_{L D}$. The volume inside the surface is kept constant and equal to $V=\frac{4 \pi}{3} r_{P}^{3} N$ with $r_{P}=2.25 \AA$. To account for the spill out of the electron density, the potential radius $r_{P}$ is chosen to be slightly higher than the Wigner-Seitz radius, $r_{S}$, for the density used in the calculation of $E_{L D}$. The parameter is adopted from ref. [30] who optimized the potential for heavy clusters. As a consequence, the spillout, which scales for our potential with $N^{1 / 3}$, is somewhat underestimated for the light clusters. Adopting nuclear terminology we call $U$ a deformed Woods-Saxon potential. The remaining parameters are $U_{0}=-6 \mathrm{eV}$ and $d=0.74 \AA$, which have been determined in ref. [30] by fitting a spherical WoodsSaxon potential to the self-consistent potentials calculated by Ekardt [2] in the framework of the Kohn-Sham approach.

\subsection{Numerics}

The so-called plateau condition is an important criterion for the Strutinsky renormalization to be valid [15]. It states that within an energy interval approximately equal to the distance between two shells $\tilde{E}$ must not depend on the smoothing parameter $\gamma$. The quality of the plateau is very good in our calculations. It is of the order of several tens of $m e V$ for heavier clusters, around $N=20$ it may amount to $0.1 \mathrm{eV} .{ }^{3}$ We choose $\gamma=3.5 N^{-1 / 3} \mathrm{eV}$ as a best value for all the cases considered.

The energies $e_{i}$ are calculated by diagonalizing $h$ in the basis of a deformed harmonic oscillator whose frequency ratio is optimized for each deformation (c.f. refs. $[28,29])$. The optimal oscillator frequency $\hbar \omega_{0}$, which governs the range of the basis wave functions, is found to be close to $3.5 \mathrm{~N}^{-1 / 3} \mathrm{eV}$ for heavier clusters. The value $7 N^{-1 / 3} \mathrm{eV}$ produces a better plateau (c.f. above) for the lighter clusters. The connection of $\hbar \omega_{0}$ to the quality of the plateau is discussed in ref. [31]. We use a basis that corresponds to roughly 16 oscillator shells in the spherical case. It ensures very good convergence for the electron spectra up to $N \approx 500$.

To save computing time in case of two-dimensional minimization the diagonalization is carried out only for the base numbers $N_{B}=35,85,115,155,205$ and 281 . The energy levels for clusters lying in between are obtained by scaling the basis spectrum according to $N$-dependence of the harmonic oscillator [32]

$$
e_{i}(N)=e_{i}\left(N_{B}\right)\left(N_{B} / N\right)^{1 / 3},
$$

This interpolation procedure has also been used in our two dimensional minimizations reported in ref. [18]. For the five-dimensional minimization there is no need for any scaling because in this case each cluster is calculated in its own potential, i.e. $N_{B}=N$.

\footnotetext{
${ }^{3}$ The plateau is more stable than for the nuclear Woods-Saxon potentials. The reason is that for neutral clusters the Fermi level lies deeper in the potential well than for nuclei (at $\approx \frac{1}{2} U_{0}$ and $\frac{4}{5} U_{0}$, respectively). This has as a consequence that the continuum of positive energies deteriorates the plateau much less in clusters than in nuclei.
} 


\section{Cluster shapes in the mass range of 10 to 310}

When displaying the potential energy surface (PES) we always locate the zero point of the energy at the liquid drop energy for spherical shape, i.e. we plot the quantity

$E_{S H}\left(\alpha, \alpha_{\mu}\right)=E\left(\alpha, \alpha_{\mu}\right)+a N-4 \pi r_{S}^{2} \sigma N^{2 / 3}=E\left(\alpha, \alpha_{\mu}\right)-\left(-1.063 N+0.654 N^{2 / 3}\right) \mathrm{eV}$.

We call it $E_{S H}$ (SH for shell), since the deviations of the energy from the spherical liquid drop value are a consequence of the valence electron shell structure.

Fig. 1 shows the valence electron levels for $N=35$ as functions of the quadrupole deformation $\alpha$. Since the shape is axially symmetric the projection of the orbital angular momentum onto the symmetry axis is conserved. We denote it by $\Lambda$. All levels with $\Lambda=0$ are two-fold degenerated (spin up and down) and all with $\Lambda \neq$ 0 four-fold (spin up and down, $\Lambda$ positive and negative). This doublet-quartet structure determines the local variation of the energy as a function of the number of valence electrons, $N$, which is equal to the number of atoms for the neutral clusters.

The Woods-Saxon levels in fig. 1 are very similar to the Clemenger's Modified Oscillator levels [4]. Up to moderate values of $\alpha$, Nilsson's deformation parameters [32] are related to ours by $\delta \approx \frac{3}{2} \alpha$ and $\epsilon \approx \alpha$. The spectrum contains a number of regions of low level density, marked with the corresponding electron numbers in fig. 1. The shapes corresponding to such "openings" in the spectrum near the Fermi level are particularly stable. These are the generalized shell closures introduced by Strutinsky $[14,15]$ into nuclear physics. From the molecular point of view the preferance of such shapes corresponds to a maximization of the HOMO-LUMO gap. The openings in the spectrum are the origin of the ondulation, which the shell correction adds to the smooth liquid drop energy. The interplay of the two contributions is illustrated in fig. 2, showing the deformation energy as a function of $\alpha$ for the three clusters with $N=72,74$ and 76 . The liquid drop energy $E_{L D}$ grows up to the scission point at $\alpha=1$ and then remains almost constant. This is expected for neutral clusters having only a surface energy but no long range Coulomb interaction between the fragments. The slight changes at large $\alpha$ are due to our shape parameterization that corresponds to deformed fragments near and after the scission point for $\alpha_{\mu}=0$ (c.f. ref. [26]). The shell energy modulates the PES substantially. There is a deformed first minimum representing the ground state of the cluster. The first minimum is caused by the $N=74$ opening at $\alpha \approx 0.3$ in fig. 1 . In addition, one sees a second minimum at larger $\alpha$. In the following we restrict the discussion mainly to the first minimum. However, we want to stress that second and third minima appear quite commonly. Most clusters tend to have strongly deformed shape-isomeric states.

It turns out that even for moderate distortions there are very often several competing shapes. It is a non-trivial task to locate the lowest minima on our fivedimensional PES. In order to find them we apply the following strategy. First we generate the two-dimensional surfaces $E_{S H}\left(\alpha, \alpha_{4}\right)$ and $E_{S H}\left(\alpha, \alpha_{3}\right)$ and find the lowest minima. These are used as the starting points of a gradient procedure to find the minima on the five-dimensional PES. 


\subsection{Results of the two-dimensional minimization}

The equilibrium deformations found for $E_{S H}\left(\alpha, \alpha_{4}\right)$ ( simultaneous minimization with respect to the quadrupole and hexadecapole deformations) are presented in fig. 1 of our first report [18]. They are also shown as open circles in figs. 10 and the upper panel of fig. 11 . In fig. 3 we show the full deformation energy $E_{S H}$ compared to the shell energy for spherical shapes. The energy gain due to deformation may amount to $2 \mathrm{eV}$. The spherical clusters with the magic numbers $N=20,40,58,92,138,198$ and 254 have an extra binding of about $1 \mathrm{eV}$ compared to the classical energy of the spherical drop. The shell closure around 260 is somewhat washed out. The reason is the $3 f$-level, lying between the 254 closure and the $4 p$-level starting the next shell at 268. This position is not a consequence of the Woods-Saxon parameterization of the potential [30]. It appears already for the spherical Kohn-Sham calculations [33].

Between the spherical shells the energy lies only about $0.1 \mathrm{eV}$ below the spherical drop value. This reflects the lower symmetry of the shape, leading to a smaller shell correction. Like nuclei, the clusters are deformed between the magic numbers because they avoid the large positive shell energy of the spherical shape.

Fig. 4 demonstrates by way of example: As a rule, a minimum of the one dimensional energy function $E_{S H}\left(\alpha, \alpha_{4}=0\right)$ belongs to the couloir around a deeper minimum in the $\alpha-\alpha_{4}$-plane. Thus, the inclusion of $\alpha_{4}$ as a second degree of freedom does not lead to the appearance of new minima. The deformation parameters $\alpha$ and $\alpha_{4}$ are strongly correlated. The contour lines enclosing the minimum have principal axes that are not parallel to the $\alpha$ or $\alpha_{4}$ axes. The energy gain due to the inclusion of $\alpha_{4}$ is typically of the order of $200-300 \mathrm{meV}$, reaching its maximum of $500 \mathrm{meV}$ for $N=154$. This is sufficient to exchange the relative positions of two minima. In fig. 4 the oblate minimum lies below the prolate one for $\alpha_{4}=0$, whereas for the equilibrium values of $\alpha_{4}$ the prolate minimum becomes the lowest. What is said about the inclusion of $\alpha_{4}$ holds also for $\alpha_{6}$. It does not lead to new minima. The energy shifts related to the relaxation in $\alpha_{6}$ are typically equal to $100 \mathrm{meV}$ or less.

On the other hand, breaking the reflection symmetry by including the octupole deformation, $\alpha_{3}$, leads to the appearance of new minima in the PES. In figs. 5 the contour plots give examples of the coexisting shape isomers. The equilibrium deformations found on the PES $E_{S H}\left(\alpha, \alpha_{3}\right)$ (simultaneous minimization with respect to the quadrupole and octupole deformations) are shown in fig. 3 of our first report [18]. For all clusters we compared the energy of the lowest minimum obtained by minimizing $E_{S H}\left(\alpha, \alpha_{3}\right)$ with the lowest minimum obtained by minimizing $E_{S H}\left(\alpha, \alpha_{4}\right)$. If the reflection asymmetric minimum was lower we considered the cluster octupole deformed.

Some selected cluster shapes obtained by two-dimensional minimization are shown in fig. 6 . The left-hand column demonstrates the shape modification due to $\alpha_{\mathbf{4}}$. The middle column shows an example of coexisting reflection symmetric and asymmetric shapes. 


\subsection{Results of the five-dimensional minimization}

The right-hand column of fig. 6 demonstrates the convergency of shape and energy when taking into account an increasing number of multipoles. Including the multipoles up to $\alpha_{6}$, the axial shape can be considered as practically relaxed. For selected examples we found the energy gain obtained by the inclusion of the multipoles $\alpha_{7,8,9,10}$ to be of the order of a few $10 \mathrm{meV}$.

We carried out the five-dimensional minimization for all clusters in the mass range of $10 \leq N \leq 90$. The results are presented in figs. 7, 8 and 9 . For the mass range of $90 \leq \mathrm{N} \leq 310$ only the even clusters have been considered. The results are presented in figs. 10,11 and 12. Due to the reflection symmetry there are always two minima with identical energy that are related by changing the signs of all $\alpha_{\mu}$ with odd $\mu$, as illustrated in fig. 5. We have fixed signs in figs. 7 and 10 such that the values of $\alpha_{3}$ are always positive. With this choice the sign of $\alpha_{5}$ may be positive or negative.

In order to find the absolute minimum we start our gradient method for all clusters from the lowest minimum found by means of the $\alpha-\alpha_{4}$ minimization. Since we do not have evidence that $\alpha_{6}$ creates any new minima the gradient search should find the lowest minimum with reflection symmetric shape. For all clusters we then compare the energy of the lowest minimum obtained by minimizing $E_{S H}\left(\alpha, \alpha_{3}\right)$ with the lowest minimum obtained by minimizing $E_{S H}\left(\alpha, \alpha_{4}\right)$. If the reflection asymmetric minimum is lower than or has a similar energy as the reflection symmetric minimum we start the gradient search also from this point. Since we do not have evidence that the inclusion of $\alpha_{5}$ and $\alpha_{6}$ creates new minima this search should provide the lowest minimum with reflection asymmetric shape. The lower one of the reflection symmetric and reflection asymmetric minima, found in this way, are included in figs. 8-12. In order to make sure that we do not end up in a local minimum (shape isomer), we started a search from the second reflection symmetric minimum ( the oblate one) for clusters with energetically competing minima. The search always ended in a local minimum with higher energy. The deformation parameters and energies of the lowest isomeric states in the lightest clusters are shown in fig. 13. Bearing this careful search in mind we believe that figs. 8-12 refer to the absolute minima of the five-dimensional PES. Since higher multipoles play an insignificant role, the axial shapes found in this way may be considered as practically relaxed. In the remainder of this section we discuss some features of these systematic calculations.

\subsection{Quadrupole deformations}

Figs. 7 and 10 show the quadrupole deformations in the upper panels. For the light clusters the results are similar to the systematics calculated for spheroidal shapes $[4,12,20]$. The shape is prolate in the lower half of a shell and oblate in the upper half. For the heavy clusters we find predominantly prolate shapes throughout the whole shell with some oblate deformation at the beginning. The dominance of prolate shapes is a well known result in nuclear physics, where it has been confirmed experimentally. According to the calculations of ref. [20] the region 
with oblate shapes at the very beginning of the shell is more extended in the heavy clusters. This may be due to the use of spherical Kohn-Sham levels instead of our Woods-Saxon potential.

The systematics of spheroidal deformations has been discussed from different points of view. Bohr and Mottelson [34] relate them to the shell structure at the frequency ratio of 2:3 in the harmonic oscillator. For small $N$ values Ekardt discusses spheroidal deformations in terms of the geometry of the $\Lambda$-substates of a spherical shell with angular momentum $l$ being filled up [12]. This discussion elucidates the transition from prolate to oblate shapes seen in the middle of the $f$-shell at $N=28$ and in the $d$-shell at $N=16$. It can be understood geometrically as a consequence of the fact that the $\Lambda=0$ states get lowest on the prolate side and highest on the oblate one. This behavior is evident from the matrix element of the spherical harmonic $Y_{20}$ which is proportional to $l(l+1)-3 \Lambda^{2}$. Geometrically the electrons are located near the poles in the $\Lambda=0$ orbitals. The extra charge will attract the ions resulting in a prolate deformation of the cluster. Hence, two electrons in the $\Lambda=0$ state drive the cluster to the prolate side, whereas two holes drive to the oblate side. For the higher $N$ many $l$-shells intertwine and this simple picture is blurred. Here a discussion in terms of classical periodic orbits is more appropriate. In ref. [35] it is pointed out that the deformations are related to the triangular and quadrangular orbits lying in the plane that contains the symmetry axis. Ref. [36] demonstrates that the preference of prolate shapes observed for the higher $N$ is explained by different changes of the length of the orbits when a sphere becomes a prolate or oblate spheroid of the same volume.

Generally a co-existence of oblate and prolate shapes is observed. This is illustrated by the contour maps shown in figs. 4 and 5 . From our calculations we cannot conclude whether the higher one is stable or becomes a saddle point if one allows for non-axial deformations. Analogous calculations for nuclei indicate that usually the higher minimum is a saddle [37]. However, the calculations for $\mathrm{Na}_{14}$ [13] show that both oblate and prolate minima are stable. There is also the possibility that the lowest minimum is weakly triaxial. For the class of ellipsoidal shapes of a pure harmonic oscillator, studied in refs. $[5,38]$ this happens frequently.

\subsection{Hexadecapole deformations}

The calculated values of $\alpha_{4}$ are shown in the second panel of figs. 7 and 10 . They change from positive values at the beginning to negative ones at the end of the shell. At the beginning of the shell the shape is lemon like, spheroidal in the middle and barrel like at the end. This is illustrated in fig. 6 (left column) showing a low-shell, mid-shell and high-shell cluster. The same tendency is found in the calculation for nuclei, for which the change of sign of $\alpha_{4}$ has been confirmed experimentally (c.f. $[14,39]$ ). In ref. [40] it is argued that the expectation value of the multipole moment $Y_{\lambda 0}$ changes sign $\left(\frac{\lambda}{2}-1\right)$ times when a spherical shell of given $l$ is being filled. Expanding the deformed potential (20) into multipoles, one sees that its expectation value will be lowered if the deformation parameter associated with the multipole $Y_{\lambda 0}$ depends on $N$ in the same way. This argument cannot be applied to 
the heavy clusters where several spherical states of given $l$ combine to one shell. A discussion in terms of periodical classical orbits does not exist.

\subsection{Octupole deformations}

The $\alpha_{3}$ values are shown in the fourth panel of figs. 7 and 10. Octupole deformations appear only for the clusters lying somewhat above a spherical shell closure. This feature is well known for nuclear equilibrium shapes $[41,42]$ and has also been found for clusters by Hamamoto et al. [43], who studied the energies for quadrupole and octupole deformation separately. Comparing the dots with the circles in the upper panel of fig. 10 one notices that at the beginning of a new shell the octupole deformation combined with a small quadrupole deformation is energetically favoured in comparision with the relatively large oblate deformations appearing if only reflection symmetric shapes are considered. Thus, taking into account the possibility of the pear-like deformation the clusters, as a rule, are prolate or octupole-deformed (with a small positive or negative quadrupole admixture), and very seldom strongly oblate. This may be partially a feature of the assumed flat bottom of the WoodsSaxon potential, since the calculations based on spherical Kohn-Sham levels [20] have a stronger tendency towards oblate deformations than our reflection symmetric calculations (c.f. ref. [18] and fig. 10, open circles). It would be interesting to see whether a more realistic radial profile of the electron potential changes the interplay between the reflection symmetric and antisymmetric multipoles.

The energy gained by octupole deformation is of the order of $200-300 \mathrm{meV}$ with a maximum of $400 \mathrm{meV}$ for $N=208$. It flattens the sharp rise of the energy $E_{S H}$ after a closed shell, as can be seen in fig. 11, also showing as open circles the energies obtained by the two-dimensional minimization with respect to $\alpha$ and $\alpha_{4}$. For the heavy clusters one should keep in mind that the energies due to shell structure and deformation are sensitive to heating.

The octupole deformation disappears abruptly, at some stage of filling the shell. Such deformation jumps are a consequence of the coexistence of relection symmetric and asymmetric minima. The jump in $\alpha_{3}$ appears when the energy of a reflection symmetric minimum falls below the energy of the reflection asymmetric one. In fact, the coexistence of distinct minima may even let the deformation jump back and forth. An example is the development of the shape above $N=40$, which is illustrated in fig. 5 . There is a competition between the "octupole" minimum at large $\alpha_{3}$ and slightly negative $\alpha$ and the "quadrupole" minimum at large positive $\alpha$, which has small or zero $\alpha_{3}$. For $N=40$ the octupole minimum is lowest, for 42 it is the quadrupole one, for 44 it is again the octupole minimum and for 46 the quadrupole minimum is lowest again, remaining the lowest one in the higher part of the shell. As will be discussed in section 4, these deformation jumps cause an even-odd staggering of the cluster binding energy. In general, shape isomerism is expected in the regions of octupole deformation.

As demonstrated by figs. 5, 7 and 10, there are strong correlations between $\alpha$ and $\alpha_{3}$. Usually, an octupole deformed minimum has also a finite quadrupole deformation. As discussed in ref. [18], this results in a finite static dipole moment 
of the cluster. Looking for these dipole moments in the microwave spectra could provide direct evidence for the predicted octupole deformations.

Some features of the $\alpha_{3}$ systematics can be understood by inspecting the electron levels as functions of $\alpha_{3}$ shown in fig. 14. In contrast to fig. 1, the spherical levels are only distorted by non-diagonal matrix elements, the most important of which are indicated by arrows in fig. 14. These are matrix elements of $Y_{30}$ between spherical electron states that differ in angular momentum $l$ by 1 ( the $\Delta l=1$ couplings) and by 3 ( the $\Delta l=3$ couplings).

Let us discuss the clusters above $N=40$ that are illustrated in fig. 5 . The $\Lambda=4$ level of the $1 g$-shell is pushed down with increasing $\alpha_{3}$, since it has a $\Delta l=1$ matrix element with the $\Lambda=4$ level of the $1 h$-shell but none with the $1 f$-shell, which does not have a $\Lambda=4$ state. Filling up this state gives rise to the octupole minimum for $N=41-44$. Since the states of maximal $\Lambda$ have a lower energy for negative $\alpha$, the shift of the octupole minimum to the oblate side is explained. The octupole deformation disappears when the gap between the $\Lambda=4$ and the upsloping level $\Lambda=3$ is crossed, i.e. at $N=46$, since $\Lambda=3$ does not favour $\alpha_{3}$.

The $\Lambda=4$ level favouring $\alpha_{3}$ has to compete with the $\Lambda=0,1$ levels that slope down in the direction of positive $\alpha$ and small $\alpha_{3}$, as can be seen in fig. 1 . Filling the $\Lambda=0,1$ levels gives rise to the quadrupole minimum in fig. 5 , which is strongly prolate deformed and represents the shape of the clusters above the octupole region. The jumps of the deformation above $N=40$ can be understood in terms of the competition between the occupation of high and low $\Lambda$ levels producing the octupole and quadrupole minima, respectively. As already discussed, $N=40$ lies in the octupole minimum. It is more beneficial to fill the next two electrons into the $\Lambda=0$ level than into $\Lambda=4$, hence the quadrupole minimum is lower for $N=42$. For the next two electrons it is better to fill the $\Lambda=4$ level, because of the gap between $\Lambda=0$ and 1. Hence the octupole minimum is lower for $N=44$. For the next two electrons it is again better to fill the $\Lambda=1$ level because of the large gap between $\Lambda=4$ and 3 . Hence the quadrupole minimum is lower and the end of the octupole region is reached.

The $2 p$-levels are pushed down by their $\Delta l=3$ coupling to the $\Lambda=0$ and 1 levels of the $1 g$-shell. This has the remarkable consequence that the magic cluster $N=40$ is octupole deformed. As seen in fig. 5 , it is very soft with respect to $\alpha_{3}$, what is reflected by the fact that the $N=40$ gap is almost independent of $\alpha_{3}$. In the five-dimensional minimization we find that the spherical shape lies only $0.05 \mathrm{eV}$ above the octupole deformed minimum. Nevertheless, this energy gain should be big enough to survive the thermal fluctuations.

Above $N=20$ the situation is quite analogous to the one above 40 . The $\Lambda=3$ level of the $1 f$-shell is pushed down by the $\Delta l=1$ coupling to the $\Lambda=3$ level from the $1 g$-shell and the $2 s$-level is pushed down by the $\Delta l=3$ coupling to the $\Lambda=0$ level from the $1 f$-shell. Again, an oblate octupole and a prolate reflection symmetric minimum compete. The energy gain by octupole deformation is somewhat less due to the lower angular momentum of the states. As a consequence, the prolate minimum lies always somewhat below the octupole one and $N=20$ is spherical, though very soft against octupole deformation. The analogous scenario also holds above $N=70$. 
Here it is the $\Lambda=5$ level from the $1 h$-shell, the $3 s$ - and, to some extent, the $2 d$ levels that are pushed down. Due to the higher angular momentum of the states the energy gain by octupole deformation is bigger and the prolate minimum now always lies slightly above the octupole one until the gap between $\Lambda=5$ and 4 in the $1 h$-shell is reached, where the prolate minimum takes over and the end of the octupole region is reached.

In conclusion, there are two mechanisms generating the octupole deformation. The $\Delta l=3$ coupling acts across the spherical shell gap leading to octupole softness or even instability for the magic clusters. The $\Delta l=1$ coupling of the states with maximal $l$ in adjacent shells pushes the $\Lambda=l$ states of the the lower shell down leading to octupole deformation for the first 4 electrons in the shell that can be accomodated into this orbit.

Systematic calculations $[41,42]$ show that nuclei are less susceptible to octupole deformation than clusters. One reason are the pairing correlations in nuclei that tend to keep the system spherical. Another one is the large spin orbit splitting in nuclei. It leads to a different pattern of octupole instability. The spin orbit potential shifts the state $j=l_{\max }+1 / 2$ into the next lower shell and the state $j=l_{\max }-1 / 2$ among the levels with lower $l$. The $\Delta l=1$ coupling is reduced, since it shifts the $j=l_{\max }-1 / 2$ levels only relative to the low $l$ levels resulting in a level redistribution but no energy gain (no shell gap opens). Thus, it is the interaction with the $j=l_{\max }+1 / 2$ levels that remains, which can only give half the energy gain. The interaction is now a combination of the $\Delta l=3$ coupling of these "intruder" level with the states of the shell they are embedded and the $\Delta l=1$ coupling to the intruders in the next higher shell. Again, the resulting repulsion is most effective at the shell beginning since the lowest states are pushed down like the $\Lambda=l$ levels in the clusters. Higher in the shell there is only a redistribution among the levels of opposite parity not generating much of a shell gap. Hence the spinorbit coupling reduces the energy gained by octupole deformation to about a half. Moreover, in nuclei the neutrons and protons may act out of phase. Nevertheless, octupole deformation is predicted and experimentally confirmed above the magic nucleus ${ }^{208} \mathrm{~Pb}[41]$.

As in the case of the quadrupole deformation, for larger $N$ many $l$-shells intertwine and the interpretation of the deformation systematics in terms of the distortion of individual $l$-shells becomes blurred. Unfortunately, a systematic discussion in terms of classical orbits, as for the quadrupole deformation, is not available for the case of the combination of octupole with small quadrupole deformations ( c.f., however, ref. [44] for the the combination of octupole with large quadrupole deformation). Bohr and Mottelson [34] and Hamamoto et al. [43] argue that if shells with $\Delta l=3$ come close together near the Fermi surface the system should be particularly apt to octupole distortions. This occurs for the shell $138<N<198$ ( $l=3$ and $6)$. Neither our calculations nor the ones given in ref. [43] show enhanced octupole deformations for this shell. Rather there is a general tendency for octupole deformation to occur at the beginning of each shell. Applying group theoretical arguments to two degenerate shells with $l=6$ and $l=3$, Hamamoto et al. [43] demonstrated the importance of non-axial octupole distortions, which is indeed born out by their 
study of the one-dimensional PES of shapes defined by combining the sphere with only one of the spherical harmonics $Y_{3 \mu}$. The correlations between these degrees of freedom and the axial and non-axial quadrupole and hexadecapole ones remain to be studied. From our investigation of the family of axial shapes it is expected that the correlations will be strong since the resulting shapes we display in figs. 9 and 12 are rather different from distortions generated by just one multipole.

Due to the tendency of the levels with maximal $\Lambda$ to prefer both octupole and negative quadrupole deformation it is likely that the oblate shapes found in ref. [20] are unstable against octupole deformation. The mean field used in that work, which is based on the spherical Kohn-Sham levels, has the increased tendency towards oblate shape compared to our Woods-Saxon potential. It is possible that this tendency will lead to many more reflection asymmetric clusters.

\subsection{The deformations $\alpha_{5}$ and $\alpha_{6}$}

Panels 3 and 5 of figs. 7 and 10 show the $\alpha_{6}$ and $\alpha_{5}$ deformations. The $\alpha_{5}$ values change their sign within each octupole region reflecting the increase of nodes with multipolarity. That has already been discussed for $\alpha_{4}$ as compared to $\alpha$ [40]. Comparison of $\alpha_{6}$ with $\alpha_{4}$ shows a more frequent crossing of the zero line. However it is not simply one node more, as could be expected from the argument given in ref. [40]. The reason is presumably the interplay between the different deformation modes that becomes increasingly important at higher multipolarity.

The $\alpha_{6}$ deformation may still give an energy gain of the order of $100 \mathrm{meV}$, as can be seen in fig. 11 from comparing the open circles with the full ones in the regions with zero $\alpha_{3}$. The same comparison in fig. 10 shows that the correlation with $\alpha_{6}$ may lead to noticeable changes of $\alpha$ and $\alpha_{4}$.

\section{Experimental consequences of cluster defor- mations}

In this section we will discuss some implications of our systematic study of the shape of $\mathrm{Na}$ clusters. It is only possible to address to selected aspects, while many more have to be left for further investigations. Here we shall not discuss ionization potentials, leaving discussion of this important experimental information for a forthcoming study that treats the Coulomb field of charged clusters within the shell correction method. As discussed in our first report [18], the combination of quadrupole and octupole deformation leads to static dipole moments that can, in principle, be detected by observing interaction with electromagnetic radiation in the microwave range. A first rough estimate of the expected dipole moments and of the wavelength of the rotational lines can be found there. A more detailed study of the dipole response in the low-frequency range will also be presented in a future paper, while the high-frequency modes will be discussed below.

In general, the magnitude of the equilibrium deformations scales with $N^{-1 / 3}$ according to general arguments concerning the degeneracy of the electron system [34]. 
The actual deformations fluctuate considerably around the expected average. Nevertheless, the scaling law sets the order of magnitue of any observable consequence of the electronic shell structure.

\subsection{Stability of the shapes}

The degree of localization of the shape in the minimum is determined by the quantal zero-point- and thermal fluctuations. The order of magnitude of the former can be roughly estimated by assuming an irrotational flow for the oscillations around the minimum. The corresponding mass coefficient $D_{\beta \beta}$ for the quadrupole deformation parameter $\beta$ can be found in ref. $[27,34]$. Taking into acoount the relation to our deformation coordinate, $\alpha \approx \sqrt{\frac{5}{4 \pi}} \beta$ ( c. f. section 3 ), the mass parameter becomes

$$
D \approx \frac{4 \pi}{5} D_{\beta \beta}=\frac{3}{10} N^{5 / 3} M r_{s}^{2}=7731 N^{5 / 3} \hbar^{2} / e V
$$

where we use $M c^{2}=23 * 939 M e V$ for the Na mass. For $N=72$ one obtains $D \approx 9.610^{6} \hbar^{2} / \mathrm{eV}$. The zero-point energy and amplitude for harmonic vibrations become, respectively,

$$
\frac{1}{2} \hbar \omega=\frac{\hbar}{2} \sqrt{C / D} \approx 1.4 m e V, \Delta \alpha^{2}=\frac{\hbar}{2} / \sqrt{C D} \approx 1.910^{-5}
$$

The numerical values correspond to $N=72$ with the curvature $C \approx 75 \mathrm{eV}$ at the minimum $\alpha=0.22$ (c. f. fig. 2). Thanks to the heavy ions the cold clusters are very well localized in their deformation minima, much better than nuclei are. (Compare the ratio $\frac{\sqrt{\Delta \alpha^{2}}}{\alpha} \approx 2.010^{-2}$ in clusters to the value of $10^{-1}$ in nuclei). This is due to the small ratio of the electron mass, which determines the stiffness $\mathrm{C}$, to the mass of the $\mathrm{Na}$ ion, which determines the mass coefficient $\mathrm{D}$. In nuclei the nucleon mass appears in both $\mathrm{C}$ and $\mathrm{D}$.

The thermal fluctuations correspond to an excitation energy of the order of $k T$, i.e., to an average potential energy of

$$
\frac{C \Delta \alpha^{2}}{2}=\frac{k T}{2}
$$

For a cluster temperature of $500^{\circ} \mathrm{K}$ the encrgy $k T \approx 45 \mathrm{meV}$ corresponds to $\Delta \alpha^{2} \approx$ 6. $10^{-4}$, which is larger then the quantal zero-point fluctuations. The situation discussed is typical for all deformation degrees of freedom considered, since the mass coefficients are comparable and the variations of the PES are, at least, in the $100 \mathrm{meV}$ range. Except for cases close to instability, the clusters are very well localized in the minima of the PES. Usually the energy difference between the ground and the isomeric configurations is equal to a few $100 \mathrm{meV}$ at least, i.e., the isomers are very weakly excited in thermal equilibrium. However, for cases with competing minima, like in the regions of octupole deformation or around $N=28$, the two shape isomers may both be thermally excited. 
The thermal stability of the shell structure was shown in ref. [34] to be controlled by the ratio

$$
\tau=\frac{2 \pi^{2} k T}{\hbar \omega_{\text {shell }}} \approx \frac{2 \pi^{2} k T}{3.5 e V N^{-1 / 3}},
$$

where $\hbar \omega_{\text {shell }}$ is the spacing between the spherical shells. ${ }^{4}$ Since deformation comes as the consequence of avoiding the large positive shell correction at spherical shape, the appearance of deformed shapes should be controlled by the same ratio $\tau$. Using $k T=45 \mathrm{meV}$ one finds $0.09,0.15$ and 0.26 for $N=20,100$ and 500 , respectively. Thus one expects that below $N=500$ the finite temperature will not significantly change the large quadrupole deformations in the middle of a shell. For the heaviest clusters considered $(N \approx 300)$, the temperature will begin to change the higher deformation modes. (The estimates of the magnitude of the energy gain for different deformation modes was given in the preceding section). In fact, the regions of spherical shape near the magic numbers will extend and the deformed regions will shrink, in accordance with the energy gain due to deformation (c.f. fig. 3).

\subsection{Splitting of the dipole resonance}

The most direct measurement of the cluster deformation is the splitting of the dipole resonance observed in the photo-absorption spectrum ( also denoted as the Mie resonance or plasmon) $[3,5,6,7,8,9,10]$. For axial shapes there are two degenerated eigenmodes in the equatorial plane and one eigenmode along the symmetry axis. In fact the observed absorption spectra show a splitting of the dipole resonance into a high-intensity peak, associated with the equatorial modes, and a low-intensity one due to the polar mode.

Assuming a spheroidal jellium model, Lipparini and Stringari [46] have derived an approximate expression for the relation between the ratio of the two resonance energies $E_{\perp}$ and $E_{\|}$

$$
\frac{E_{\perp}}{E_{\|}}=\left(1+\frac{1}{5} \delta\right) /\left(1-\frac{2}{5} \delta\right)
$$

with the deformation parameter defined as

$$
\delta=\frac{3}{2} \frac{R_{\|}^{2}-R_{\perp}^{2}}{R_{\|}^{2}+2 R_{\perp}^{2}} .
$$

Using eqs. (27) and (19) one finds that

$$
\alpha=\frac{\delta}{1+\delta / 3}
$$

Inverting eq. (27) we derive the "experimental" values for $\delta$, which we compare with our calculated $\alpha$ 's. One may derive the same expression starting from the

\footnotetext{
${ }^{4} \mathrm{~A}$ very close criteria was formulated in ref. [45], where the critical temperature for the shell disappearence was introduced as $k T_{s}=\frac{1}{\pi} \sqrt{\frac{3}{2}} h \omega_{\text {shell }}$.
} 
splitting of the classical Mie resonance of an ellipsoidal metal body. ${ }^{5}$

Fig. 15 compares the experimental values of $\delta$ obtained from the experimental ratios of the energies of the two peaks in the photo-absorption spectrum by using expression (27) with the calculated $\delta$ values. As already pointed out in our first paper [18], the calculations reproduce the experimental trends very well. Both in the $1 d$-shell and in the $1 f-2 p$-shell the experiments are in accordance with the calculated transition from prolate to oblate shape around $N=16$ and $N=30$, respectively. This transition has also been found in calculation within the restricted family of spheroidal shapes $[12,20]$. Hence, the lifting of the restriction in the shapes does not change the occurrence of the prolate-oblate transition in these two shells.

Like for the family of $\alpha, \alpha_{4}$-shapes studied in our first report [18], we find the prolate and oblate shapes coexisting for the clusters $N=27$ and 28 at nearly the same energy. It is interesting that the Copenhagen group finds two peaks of equal intensity [8] just for these electron numbers. The equal intensity may be understood if the clusters occupy the prolate and the oblate minima with equal probability, as expected for nearly equal energies $[8,18]$. Another possibility of coexistence beween oblate and prolate shapes appears for $N=12$ (c.f. fig. 13). In ref. [6] the spectra show two humps of about the same height. However, the experimental picture is less clear, since the absorption cross section increases again above the second peak. This has been interpreted by the authors as evidence for a third peak that might be due to a triaxial shape. As mentioned before, our calculations cannot answer the question whether there is a barrier beween the two minima or not.

Above $N=40$ there is a discrepancy between our calculations and the experiments, which indicate oblate shapes for $N_{e}=41-48[8,18]$. Combining all the multipoles, we obtain strong prolate deformation for $N=42$ and $N=45-48$ and for $N=43$ and 44 an octupole deformation combined with a slight oblate quadrupole deformation. The reflection asymmetric shape might account qualitativly for the observed small high-frequency peak. However, there is the problem that this minimum has almost the same energy as the prolate reflection symmetric one. The close energy of the two minima is refected by the jumping of the shape in this region, as seen in figs. 5 and 12 . Comparable probability of the two shapes should lead to two peaks of about the same height, as seen near $N=27$. Presently it is not clear, whether this discrepancy reflects the property of the Woods-Saxon potential of being more prone towards prolate shape than real clusters ( The calculations [20] based on spherical Kohn-Sham levels tend to give more oblate clusters. However this result may be changed if higher multipoles of the shape are taken into account. c. f. discussion in sect. 3.5). It is also possible that the collective interpretation of the resonance splitting is not appropriate because of a significant fragmentation of the dipole strength among the different particle-hole excitations. Clearly, this problem can only be resolved by a microscopic calculation of the dipole response function for deformed clusters. Such studies could also shed light on the question as to how well the ratio of the resonance energies is determined by expression (27),

\footnotetext{
${ }^{5}$ Kresin has demonstrated that also for small clusters the frequency ratio is very close to the classical one [47].
} 
which is derived under the assumption of spheroidal shape. The assumption that the frequency ratio is controlled mainly by the ratio $\frac{R_{l}}{R_{\perp}}$ can be supported by angular momentum coupling arguments $[34,48]$. However, a more quantitative study is neccessary.

Most of the experiments shown in fig. 14 correspond to singly charged clusters. We assume that the shapes of these clusters are the same as for neutrals. This can be justified by the fact that the shape is mainly determined by shell effects, which are almost the same for neutrals and for singly charged clusters, since they are not very sensitive to the depth of the average electron potential. ${ }^{6}$

\subsection{Separation energies}

Fig. 3 demonstrates the drastical effects that the cluster deformation has on the binding energies. The large positive shell correction appearing in the middle of the spherical shell is completely avoided in going to non-spherical shapes. More detailed pictures of the binding energies including all axial deformations are given by figs. 8 and 11. Comparing these calculations with the calculations of ref. [20], which consider only spheroidal cluster deformation, and our two-dimensional calculations [18] shown as open circles in fig. 11, one notices that the more flexible the family of shapes is, the smoother the $N$-dependence of the energy becomes. The richer variety of shapes cuts away the spikes in the deformed region just as the spheroidal deformation cuts the huge spherical shell maxima away (c.f. fig. 3). A direct comparison of the binding energies with the experimental values of the derivative of the abundances, as suggested in ref. [20], seems problematic to us since cluster abundances are most likely to be related to separation energies. Comparing fig. 11 with fig. 5 of ref. [20] one notices that the most pronounced open shell structures of ref. [20] ( at $N=72,112,172$ and 236) seem to be also present for our more general shapes, though they appear at slightly different values of $N$.

Another conspicuous feature of the binding energies is the doublet-quartet structure that emerges from the two-fold and four-fold degeneracy of the $\Lambda=0$ and $\Lambda \neq 0$ electron states. This structure is an immediate consequence of the assumed axial shape. In fig. Sa the quartets show up in binding energies as inverted parabolas consisting of five points or in the separation energies, in fig. 8b, as upsloping straight lines consisting of four points. The experimental abundances [50] as well as the experimental separation energies [51] shown in fig. 16 provide some evidence for such quartets starting with $N=15,27$ and 31 , related to the $\Lambda=2$ ld-electron state and to the $\Lambda=2$ and 3 levels of the $1 f$-state, respectively. Pedersen et al. [50] have also found some evidence for $N=47,51$ and 55 presumably related to the $\Lambda=2,3$ and $4 \mathrm{lg}$-levels. The $N$-dependence within a quartet is easy to understand. Filling

\footnotetext{
${ }^{6}$ The main difference between the sigly charged and neutral clusters seems to be a larger fragmentation (Landau damping) in the neutrals, which has been explained by Yannouleas et al. [49]. Accordingly many of the experimental absorption spectra for neutrals do not show the simple two-peak structure (c.f. ref. [6]) but rather shallow structures that cannot be interpreted in terms of the simple collective picture employed here.
} 
electrons into the same $\Lambda$-level results in a quadratic growth of the energy

$$
E(N)=E_{o}+\left(N-N_{o}\right) e-\frac{\left(\left(N-N_{o}\right) \frac{d e}{d \alpha}\right)^{2}}{2 C}
$$

where $E_{o}$ and $N_{o}$ are the energy and electron number of the last cluster before the quartet starts. The formula assumes that the relative energy of the quartet members can be obtained from the approximate deformation energy

$$
E(\alpha)=E_{o}+\frac{C}{2}\left(\alpha-\alpha_{o}\right)^{2}+\left(N-N_{o}\right)\left(e+\frac{d e}{d \alpha}\left(\alpha-\alpha_{o}\right)\right),
$$

which is correct if the polarization of each electron in the quartet level at the energy $e$ is small enough for only the leading order terms in the deformation parameter $\alpha$ to be important. ${ }^{7}$ The quartets $N=11,23$ and 47 in figs. 8 and 15 show this $N$ dependence clearly.

The experimentally observed quartets are perturbed by an overlaying even-odd staggering. The origin of this staggering is not clear at the moment. It may indicate a deviation from axial shape that would lift the four-fold degeneracy or it could be due to correlations between the electron spins according to Hund's rule as in atoms. However, within our approach, restricted to axial shapes and no spin correlations, there is also a mechanism that may perturb quartets. Take as an example, $N=27,28,29$ and 30 , which, according to fig. 1 , is the $\Lambda=2$ quartet on the prolate side. For $N=27$ the shape is prolate, whereas for $N=28$ an oblate shape is preferred. This shape change leads to a gain relative to the energy given by eq. (30). For $N=29$ the cluster goes back to the prolate side $(\Lambda=2$ and 3 are filled and the gap to $\Lambda=1$ disfavours oblate shape) and thus back to the energy (30), where it remains for $N=30$. The resulting $N$-dependence of the binding energy displays an even-odd staggering. In a similar way the quartets starting at $N=15,31$ and 41 are perturbed by jumps in the cluster shape. In our calculation, there is also a competing shape for the $N=11$ quartet (c.f. fig. 13), which however lies a little too high in the calculations to perturb this quartet. It is interesting to note that for the quartets $N=11,15$ and 27 there is independent evidence for about equal energy of the prolate and oblate shape from the splitting of the dipole resonance (c.f. discussion in the preceding section).

Fig. 16 shows that the calculated separation energies reproduce rather well the magnitude of the experimental ones. This means that one may estimate the smooth trend of the separation energies of the very small systems considered by using the bulk surface tension and evaporation enthalpy in eq.(22) without the inclusion of a curvature term. It remains to be seen what the consequences of an extra charge are, since the experiments are done for singly charged ions. Since the experiments are sensitive to energy differences between ions of the same charge state, most of the Coulomb energy is expected to cancel.

The calculated shell structure that modulates the smooth trend seems to be correlated with the experimental modulation, though it is too pronounced. The

\footnotetext{
${ }^{7}$ The argument can easily be generalized to many deformation parameters resulting still in the same $N$-dependence given by eq. (30).
} 
inclusion of finite temperature will not damp the shell structure significantly, since the cluster temperature of $\sim 50 \mathrm{meV}$ is much smaller than the typical subshell structure of the light clusters, that is $\sim 1 \mathrm{eV}$ (c.f. section 4.1). Thus, if the analysis of the experimental evaporation spectra does not underestimate the shell fluctuations, there must be additional mechanisms to damp the shell structure. These could be the appearance of non-axial deformations $[6,13]$ or the roughness of the surface caused by the discrete ionic background [52].

Another feature of the data in fig. 16 is the systematic even-odd staggering. The calculations show the even-odd structure, if $\Lambda=0$ electron levels are occupied or the quartets are perturbed by jumps of the shape, as discussed above. The $N=11$ and $N=23$ quartets remain clearly visible in the calculations whereas there is no evidence for them in the data (though at $N=23$ there might be the beginning of a perturbed quartet). The data seem to indicate a distorted quartet starting at $N=31$, in agreement with the abundance data of Pedersen et al. [50]. If this interpretation is correct, it would indicate that the $\Lambda=0$ level from the $2 p$-state lies above the $\Lambda=3$ level from the $1 f$-state rather then below it, as in fig. 1 for $\alpha>0.2$.

\section{Conclusions}

The shell correction method is an effective calculation scheme permitting the study of the cluster shape within a multi-dimensional family of axial shapes. A wide range of clusters up to mass 300 has been studied and an extension to mass 1000 is well within the reach of the method. Strong deviations from the spheroidal shape are found. The size of the deformation parameters scales with $N^{-1 / 3}$, however significant fluctuations around this scaling law appear in the individual clusters. There are strong correlations between the different deformation parameters of the considered family of shapes, and this is expected to be the case for other shape parameterization as well. The lightest clusters below mass 50 take shapes which remind that of big molecules (c. f. fig. 9). This is remarkable since the calculations use the jellium approximation for the ions. The shapes reflect the spatial distribution of the delocalized valence electrons. For the heavier clusters the shapes are more rounded, still they are far from being spheroidal. At the very beginning of each shell clusters have a tendency to look pear-like. In the higher part of the shell they prefer reflection symmetric shapes looking lemon-like in the lower and barrel-like in the upper half of the shell. Though we did not study the question in detail, the calculations reveal the existence of many isomeric shapes.

Direct experimental information about the cluster shapes is very restricted. So far it is mainly the splitting of the dipole resonance that contains such information. The measurements are consistent with our calculations, except for some clusters above mass 40 . In particular, the cases with the two peaks of equal height seem to be correlated with the calculated coexistence of prolate and oblate shapes. Clearly, it would be interesting to measure heavier clusters. Another open problem, mostly a theoretical one, is the relation between the shape of the clusters and the resonance splitting. We used a rather qualitative relation, which is based on the assumption 
of spheroidal shape. It is necessary to study the dipole resonance on a microscopic basis to see the influence of the more complicated shapes. One will also have to take into account the fragmentation of the resonance among the particle-hole excitations.

Compared to calculations that assume a spherical shape, the cluster deformation strongly modifies the binding and separation energies as functions of the number of atoms in the cluster. There are only few measurements that provide separation energies for comparison. They coincide moderatly well with our calculated values, demonstrating the ability of the shell correction method with deformations to provide the separation energies. The calculated shell structure seems still to be somewhat too pronounced. The calculated energies show a doublet-quartet structure, which is a consequence of the assumed axial shape. The experiments provide some evidence for the quartets, which are perturbed by an overlaying even-odd staggering. Jumps of the axial shape as a function of the number of atoms generate such staggering for some of the calculated quartets. It would be highly desirable to relate the rich data on cluster abundances to the calculated separation energies in a more quantitative way.

The authors wish to express their deep gratitude to S. Bjørnholm for his great encouragement and the numerous clarifying discussions. We would also like to thank J. Borggreen and J. Pedersen for providing us with experimental results prior to publication. 


\section{References}

[1] W.D. Knight, K. Clemenger, W.A. de Heer, W.A. Saunders, M.Y. Chou, M.L. Cohen, Phys.Rev.Lett. 52 (1984) 2141.

[2] W. Ekardt, Phys. Rev. B29 (1984) 1558.

[3] W.A. de Heer, W.D. Knight, M.Y. Chou, M.L. Cohen. Solid State Phys. 40 (1987) 93.

[4] K. Clemenger, Phys. Rev. B32 (1985) 1359.

[5] K. Selby, M. Vollmer, J. Masui, V. Kresin, W. de Heer, W. Knight, Phys. Rev. B40 (1989) 5417.

[6] K. Selby, V. Kresin, J. Masui, M. Vollmer, W. de Heer, A. Scheidemann, W. Knight, Phys. Rev. B43 (1991) 4565.

[7] C. Bréchignac, Ph. Cahuzac, F. Carlier, M. de Frutos, J. Leygnier, Chem. Phys. Lett. 189 (1992) 28

[8] J. Borggreen, P. Chowdhury, N. Kebaïli, L. Lundsberg-Nielsen, K. Lützenkirchen, M. B. Nielsen, J. Pedersen, H. D. Rasmussen, Phys. Rev. B48 (1993) 17507

[9] J. Tiggesbäumker, L. Köller, H. O. Lutz, K. H. Meiwes-Broer, Chem. Phys. Lett. 190 (1992) 42

[10] S. Pollack, C. R. C. Wang, M. M.Kappes, J. Chem. Phys. 94 (1991) 2496

[11] A. Tamura, T. Ichinokowa, Surf. Sci. 136 (1984) 437.

[12] W. Ekardt, Z. Penzar. Phys. Rev. B38 (1988) 4273.

[13] G. Lauritsch, P.G. Reinhard, J. Meyer, M. Brack, Phys. Lett. A160 (1991) 179.

[14] M. Brack, J. Damgaard, A.S. Jensen, H.C. Pauli, V.M. Strutinsky, C.Y. Wong, Rev. Mod. Phys. 44 (1972) 320.

[1.5] V.M. Strutinsky, Nucl. Phys. A95 (1967) 420; Nucl. Phys. A122 (1968) 1.

[16] M. Nakmura, Y. Ishi, A. Tamura, S. Sugano, Phys. Rev.A42 (1990) 2267

[17] S. Sugano, Microcluster Phisics, (Springer, Berlin, Heidelberg, 1991)

[18] S. Frauendorf, V.V. Pashkevich, Z. Phys D26 (1993) S 98

[19] C. Yannouleas, Uzi Landman, Phys. Rev. B 48 (1993) 8376

[20] S.M. Reimann, M. Brack, K. Hansen, Z. Phys. D28 (1993) 235 
[21] P. Ballone, W. Andreoni, R. Car, M. Parinello, Europhys. Lett. 8 (1989) 73 A. Bulgac, D. Kusnezov, Phys. Rev. Lett. 68 (1992) 1335

[22] T. P. Martin, T. Bergmann, H. Göhlich, T. Lange, Chem. Phys. Lett. 172 (1990) 209, Z. Phys. D19 (1991) 25

[23] C. Kittel, Introduction to Solid State Physics, (Wiley, New York, 1976); German translation: Einführung in die Festkorperphysik, (München, Wien, Oldenbourg, 1977).

[24] Landolt-Börnstein, Zahlenwerte und Funktionen aus Physik, Chemie, Astronomie, Geophysik und Technik, (Springer-Verlag) V IV/2, p.250 ff.

[25] International Critical Tables (McGraw 1926) Vol. I, p. 102.

[26] V.V. Pashkevich, Nucl. Phys. A169 (1971) 275.

[27] N. Bohr and J.A. Wheeler, Phys.Rev. 56 (1939) 426.

[28] V.V. Pashkevich and V.M. Strutinsky, Yad. Fiz. 9 (1969) 56.

[29] J. Damgaard, H.C. Pauli, V.V. Pashkevich and V.M. Strutinsky, Nucl.Phys. A135 (1969) 432.

[30] H. Nishioka, K. Hansen, B.R. Mottelson, Phys. Rev. B42 (1990) 9377.

[31] V.V. Pashkevich, Nucl. Phys. A477 (1988) 1.

[32] S.G. Nilsson Mat. Fys. Medd. Dan. Vid. Selsk. 29 no. 16.

[33] M. Brack, O. Genzken, K. Hansen Z. Phys. D21 (1991) 65.

[34] A. Bohr, B. R. Mottelson, Nuclear Structure (Benjamin, New York,1975) Vol. II.

[35] V.M. Strutinsky, A.G. Magner, S.R. Ofengenden, T. Doessing, Z. Phys. A283 (1977) 269.

[36] H. Frisk, Nucl. Phys. A511 (1990) 309.

[37] D.A. Arseniev, L.A. Malov, V.V. Pashkevich, V.G. Soloviev, Izv. Akad. Nauk SSSR, Ser. Fiz. 32 (1968) 866.

[38] Y. Takahashi, to be published.

[39] F.A. Gareev, S.P. Ivanova, V.V. Pashkevich, Yad. Fiz. 11 (1970) 1200.

[40] G. F.Bertsch, Phys. Lett. B26 (1967) 130

[41] S. Åberg et al., Ann. Rev. Nucl. Part. Sci. 40 (1990) 439. 
[42] V.V. Pashkevich, Proc. Int. School-Seminar on Heavy Ion Physics, Alushta (1983); Dubna, JINR, D7-83-644, p. 405.

[43] I. Hamamoto, B.R. Mottelson, H.X. Xie, X.Z. Zhang. Z. Phys. D21 (1991) 16

[44] K. Arita, K. Matsuyanagi, Prog. Theoretical Phys., 89 (1993) 389

[45] V.M. Strutinsky and V.M. Kolomiets, Proc. of the Eight Winter School of the Leningrad Institute of Nuclear Physics on the Nuclear Physics and Physics of Elementary Particles. Leningrad (1973) p.483-594.( In Russian ).

[46] E. Lipparini, S. Stringari, Z. Phys. D18 (1991) 193

[47] V. Kresin, Phys. Rep. 220 (1992) 1

[48] J. M. Pacheco, R. Broglia, B. R. Mottelson, Z. Phys. D21 (1991) 289

[49] C. Yannouleas, R. A. Broglia, M. Brack, P. F. Bortignon, Phys. Rev. Lett. 63 (1989) 255

[50] J. Pedersen, priv. communication

[51] C. Bréchignac, Ph. Cahuzac, J.Leygnier, R. Pflaum, J.Ph. Roux and J. Weiner, Z. Phys. D12 (1983) 199.

[52] N. Pavloff and M. S. Hansen, Z. Phys. D24 (1992) 75 


\section{Figure Captions}

Fig. 1. Electron levels as functions of the deformation parameter $\alpha$. The calculation corresponds to the Woods-Saxon well for $N=35$. Levels with positive (negative) parity are drawn by full (dashed) lines. The numbers indicate the angular momentum projection $\Lambda$ onto the symmetry axis. The numbers obtained by filling electrons into the level are given in circles. The quantum numbers for the spherical well are given at $\alpha=0$.

Fig. 2. Deformation energies for the clusters $N=72,74$ (shifted by $2 \mathrm{eV}$ ) and $N=76$ (shifted by $4 \mathrm{eV}$ ). The shapes are pure Cassinian ovaloids, which break into two fragments at $\alpha=1$. The full and dashed lines denote $E_{S H}$ and $E_{L D}$, respectively.

Fig. 3. Deformation energies at the first minimum after simultaneous minimization with respect to $\alpha$ and $\alpha_{4}$ (open circles). The shell energies for spherical shapes are included (full circles).

Fig. 4. Energy for $N=44$ as a function of the deformation parameters $\alpha$ and $\alpha_{4}$. Each contour line corresponds to $\Delta E_{S H}=0.1 \mathrm{eV}$. Minima (maxima) are labelled by crosses (dots).

Fig. 5. Energy for $N=40,42,44$ and 46 as a function of the deformation parameters $\alpha$ and $\alpha_{3}$. Each contour line corresponds to $\Delta E_{S H}=0.1 \mathrm{eV}$. Minima (maxima) are labelled by crosses (dots).

Fig. 6. Examples of cluster shapes. The left column shows typical reflection symmetric ground state shapes, which are calculated by minimizing the two-dimensional PES $E\left(\alpha, \alpha_{4}\right)$. The upper cluster $\left(N=150, \alpha=0.209, \alpha_{4}=0.095\right)$ is at the begining of the shell, the middle one $\left(N=166, \alpha=0.218, \alpha_{4}=0.000\right)$ is at the mid-shell and the lower one $\left(N=184, \alpha=0.095, \alpha_{4}=-0.080\right)$ is in the upper part of the shell. The middle column shows three coexisting shapes for $N=70$ calculated by minimizing the two-dimensional PES $E\left(\alpha, \alpha_{3}\right)$. The middle is the ground state $\left(\alpha=0.022, \alpha_{3}=0.169, E_{S H}=-0.18 \mathrm{eV}\right)$, the top is the first shape iso$\operatorname{mer}\left(\alpha=-0.26, \alpha_{3}=0.000, E_{S H}=0.14 \mathrm{eV}\right)$ and the bottom is the second isomer $\left(\alpha=0.241, \alpha_{3}=0.000, E_{S H}=0.20 \mathrm{eV}\right)$. The right column demonstrates the strategy and convergency of the minimization on the five-dimensional PES for $N=210$. The top $\left(\alpha=0.164, \alpha_{4}=0.087, E_{S H}=-0.049 \mathrm{eV}\right)$ is lowest minimum of the twodimensional PES $E\left(\alpha, \alpha_{4}\right)$. The middle $\left(\alpha=0.088, \alpha_{3}=0.103, E_{S H}=-0.228 \mathrm{eV}\right)$ is the lowest minimum of the two-dimensional PES $E\left(\alpha, a l_{3}\right)$. The bottom $(\alpha=$ $\left.0.1104, \alpha_{3}=0.0995, \alpha_{4}=0.0385, \alpha_{5}=0.0319, \alpha_{6}=0.0041, E_{S H}=-0.378 \mathrm{eV}\right)$ is the minimum on the five-dimensional PES found by starting from the shape in the middle. Starting from the shape on the top results in a local minimum with higher energy.

Fig. 7. Deformation parameters of the Na clusters in the mass range $10 \leq N \leq 92$. 
Fig. 8. The shell part of the binding energies, $E_{S H}$, (upper panel) and monomer separation energies $\Delta_{1} E(N)=E_{S H}(N)-E_{S H}(N+1)$ of the Na clusters in the mass range $10 \leq N \leq 90$. Only the modulation due to the shell structure is depicted. The total binding and separation energies are to be obtained by adding the energy of the spherical liquid drop, c.f. eq.(22).

Fig. 9. Shapes of the $N$ a clusters in the mass range $10 \leq N \leq 89$. The clusters are ordered according to their electron number $N$ from the left to the right. The values of $N$ are given for the clusters on the outer border of the figure. The symmetry axes lie horizontally.

Fig. 10. Deformation parameters of the even $\mathrm{Na}$ clusters in the mass range $50 \leq N \leq 310$. The results of two-dimensional minimization with respect to $\alpha$ and $\alpha_{4}[18]$ are included as open circles.

Fig. 11. The shell part of the binding energies $E_{S H}$ (upper panel) and half of the dimer separation energies $\Delta_{1} E(N)=\left(E_{S H}(N)-E_{S H}(N+2)\right) / 2$ of the even $\mathrm{Na}$ clusters in the mass range $50 \leq N \leq 310$. Only the modulation due to the shell structure is depicted. The total energies are to be obtained by adding the energy of the spherical liquid drop eq.(22). The open circles in the upper panel show the results of two-dimensional minimization with respect to $\alpha$ and $\alpha_{4}$ [18]. The open circles in the lower panel show half of the dimer separation energies for the sphere.

Fig. 12. Shapes of the even $\mathrm{Na}$ clusters in the mass range $90 \leq N \leq 310$. The clusters are ordered according to their electron number $N$ from the left to the right. The values of $N$ are given for the clusters on the outer border of the figure. The symmetry axes lie horizontally.

Fig. 13. Binding energies $E_{S H}$ and deformation parameters for the ground states and the lowest isomeric states in the lightest clusters. Different types of symbols permit associating the isomers in the panels. With ascending energy the following symbols are used : full circle, open circle, square, star and u-cross. The scale of the middle panel on the right side shows the deformation parameter $\delta$ used in eq.(27) to describe the splitting of the dipole resonance, which is related to $\alpha$ by eq.(29).

Fig. 14. Electron levels as functions of the deformation parameter $\alpha_{3}$. The calculation corresponds to the Woods-Saxon well for $N=35$. The numbers indicate the angular momentum projection $\Lambda$ onto the symmetry axis. The numbers obtained by filling electrons into the levels are given in circles. The quantum numbers for the spherical well are given at $\alpha=0$. The arrows indicate the octupole couplings between the spherical states, where $\Delta l$ is quoted.

Fig. 15. Experimental $\delta$ values (stars) obtained from the splitting of the dipole resonance by means of eq.(27) and calculated $\delta$ values obtained by means of the 
relation (29) from the $\alpha$ values shown in fig.s 7 and 13. Low lying isomers are included, where the same use of symbols is made as in fig. 13, i. e. with ascending energy full circle, open circle, square and star are used. The data are taken from ref. [8] where singly charged clusters were studied. The point for $\mathrm{N}=10$ and is from ref. [6], where neutrals were studied. No errors have been estimated for this case.

Fig. 16. Experimental separation energies obtained in ref. [51] for singly charged clusters (dashed line) compared with the calculated ones (full line). The experiments only permit to obtain the mean value $\left(\Delta_{1} E(21)+\Delta_{1} E(22)\right) / 2$, which is shown as the horizontal bar. The thick solid line represents the smooth separation energy of spherical clusters, as given by eq.(22). 



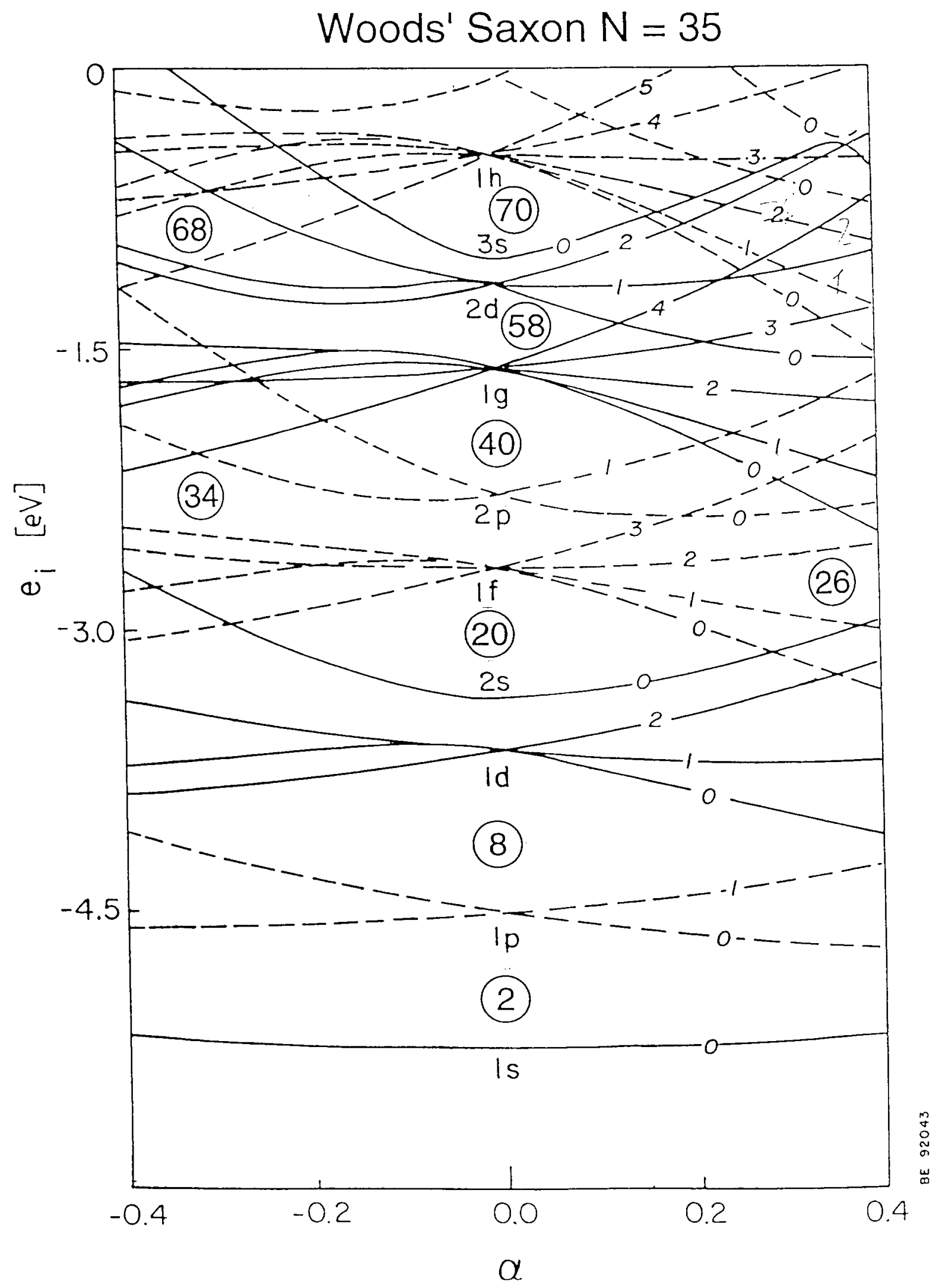

Fig. 1 


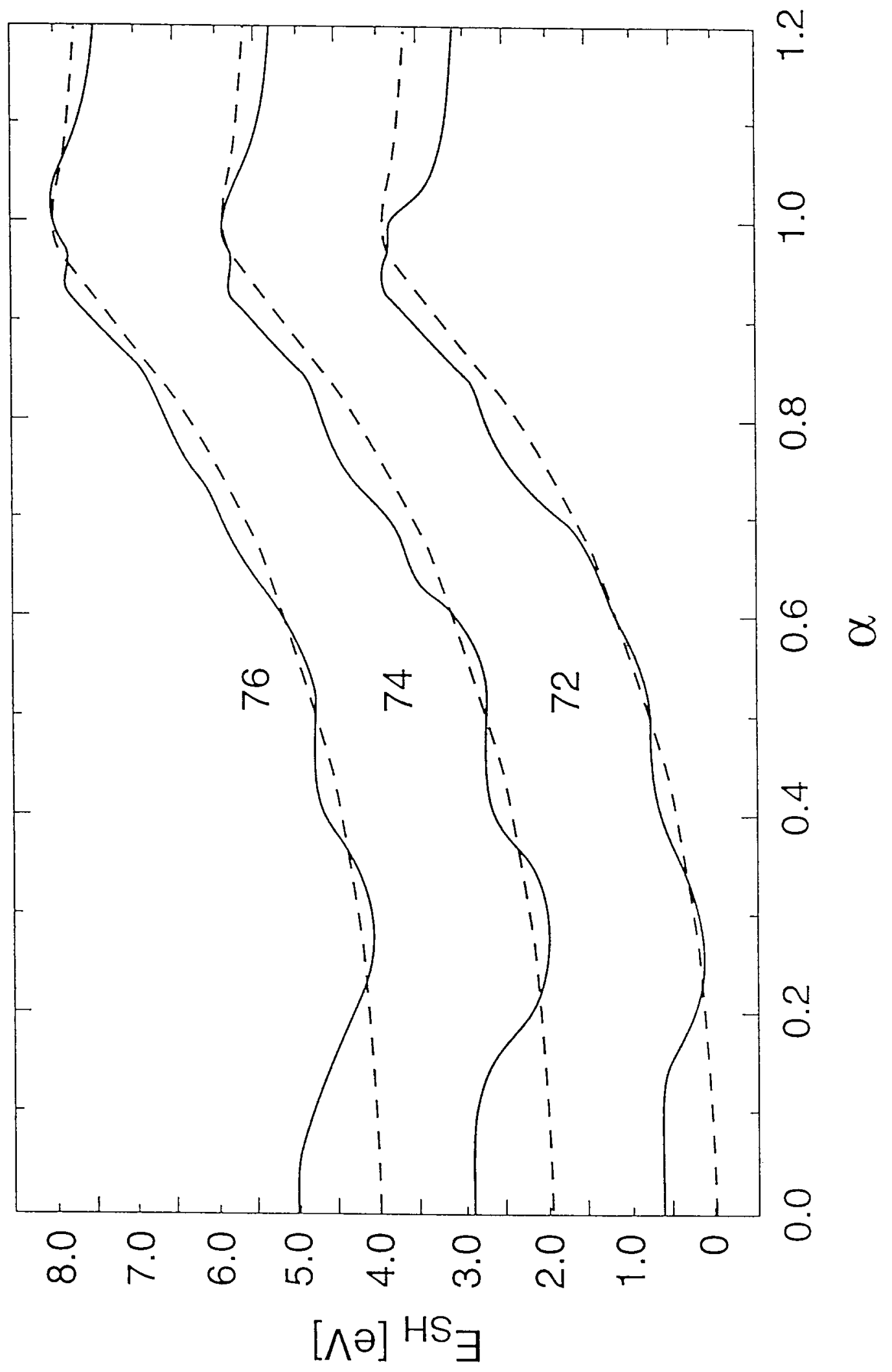

Fig. 2 


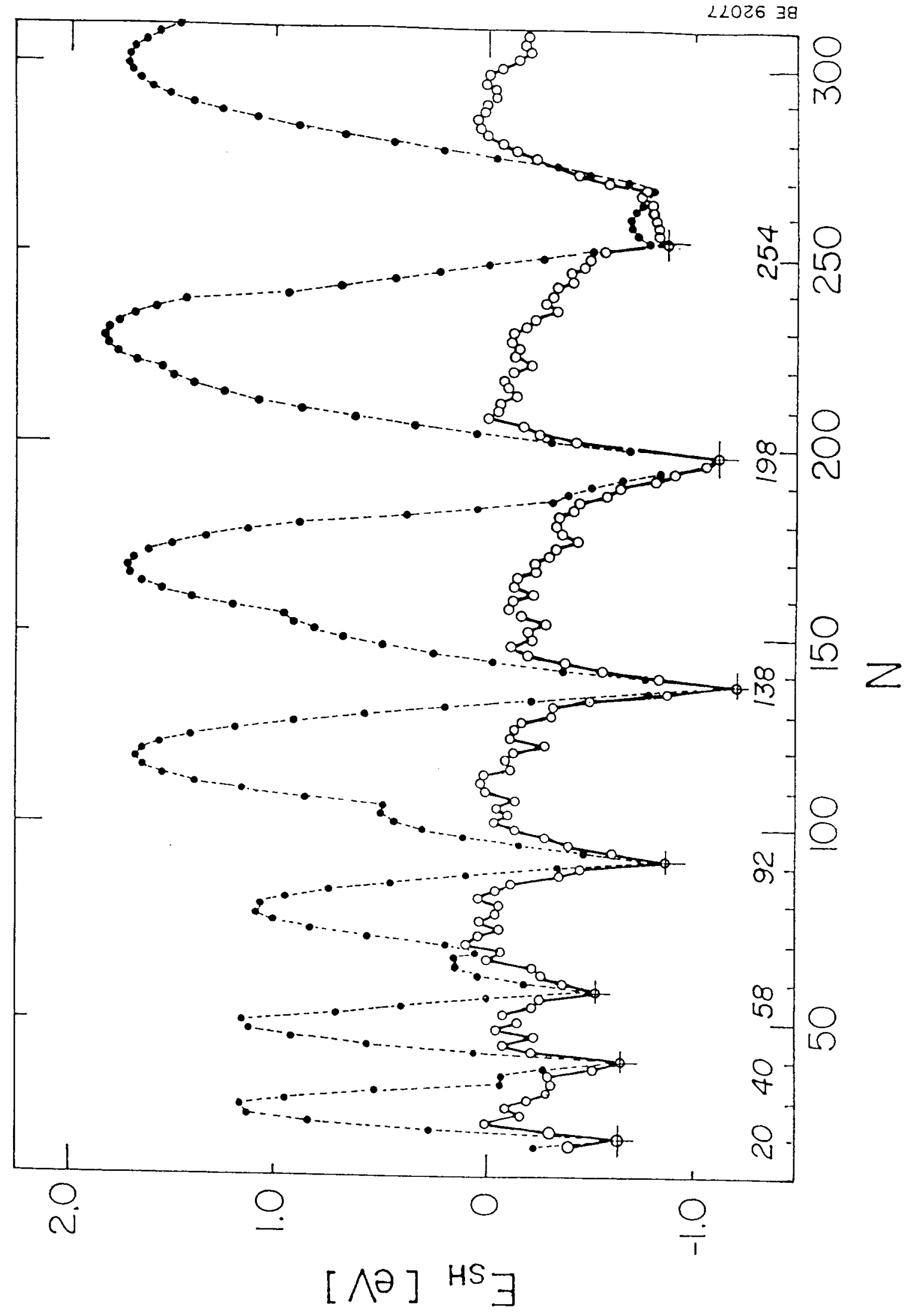

Fig. 3 


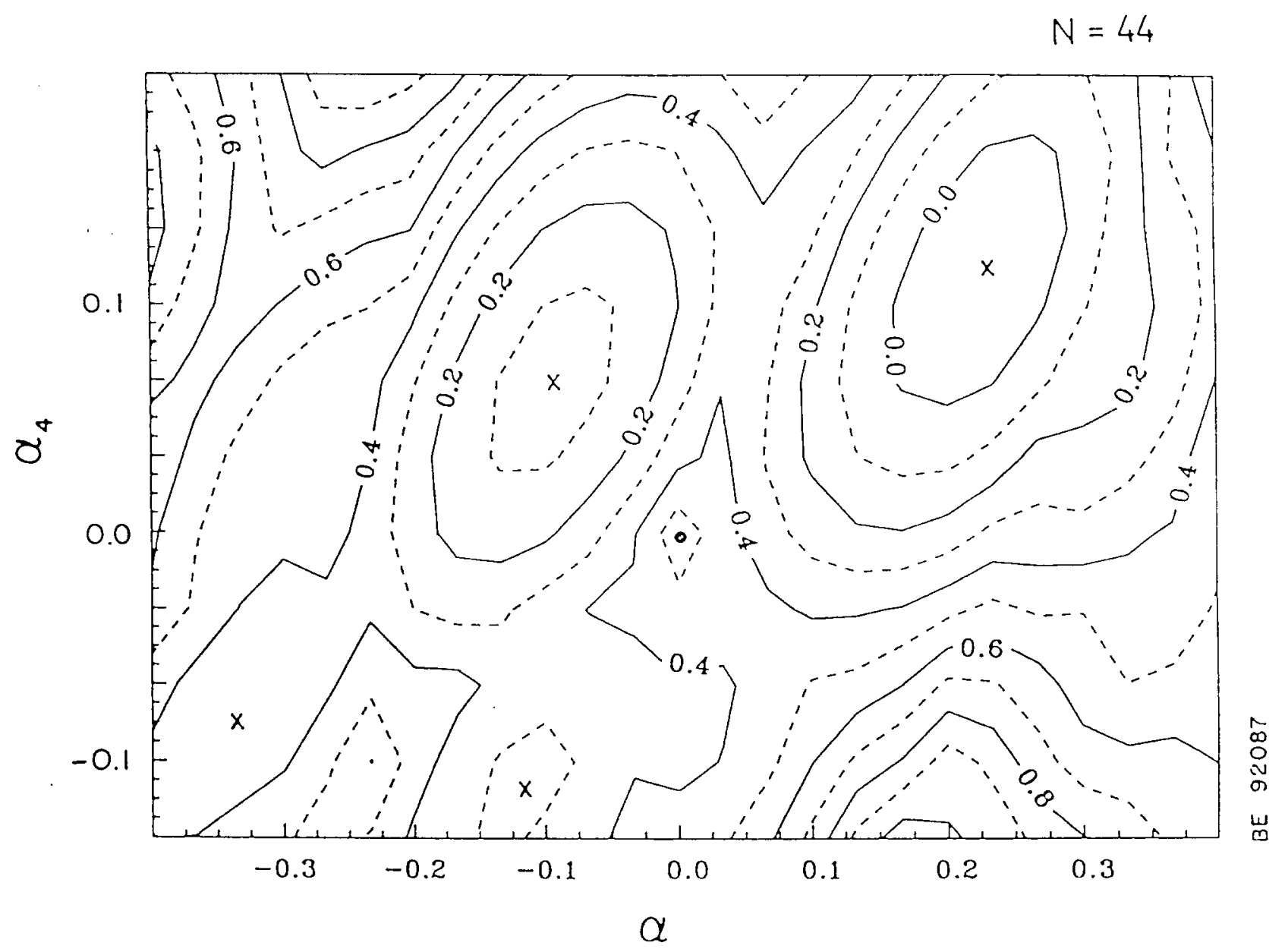

Fig. 4 


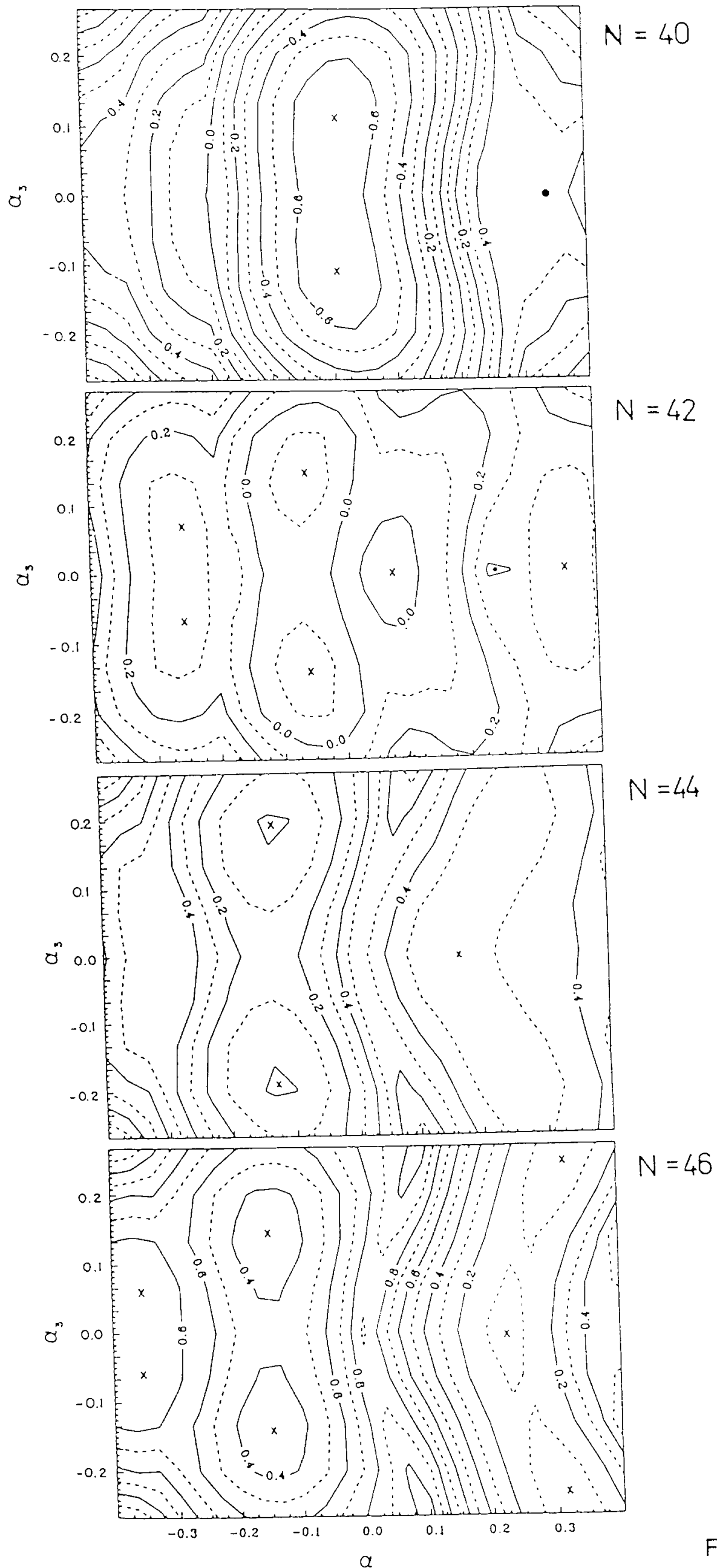

Fig. 5 

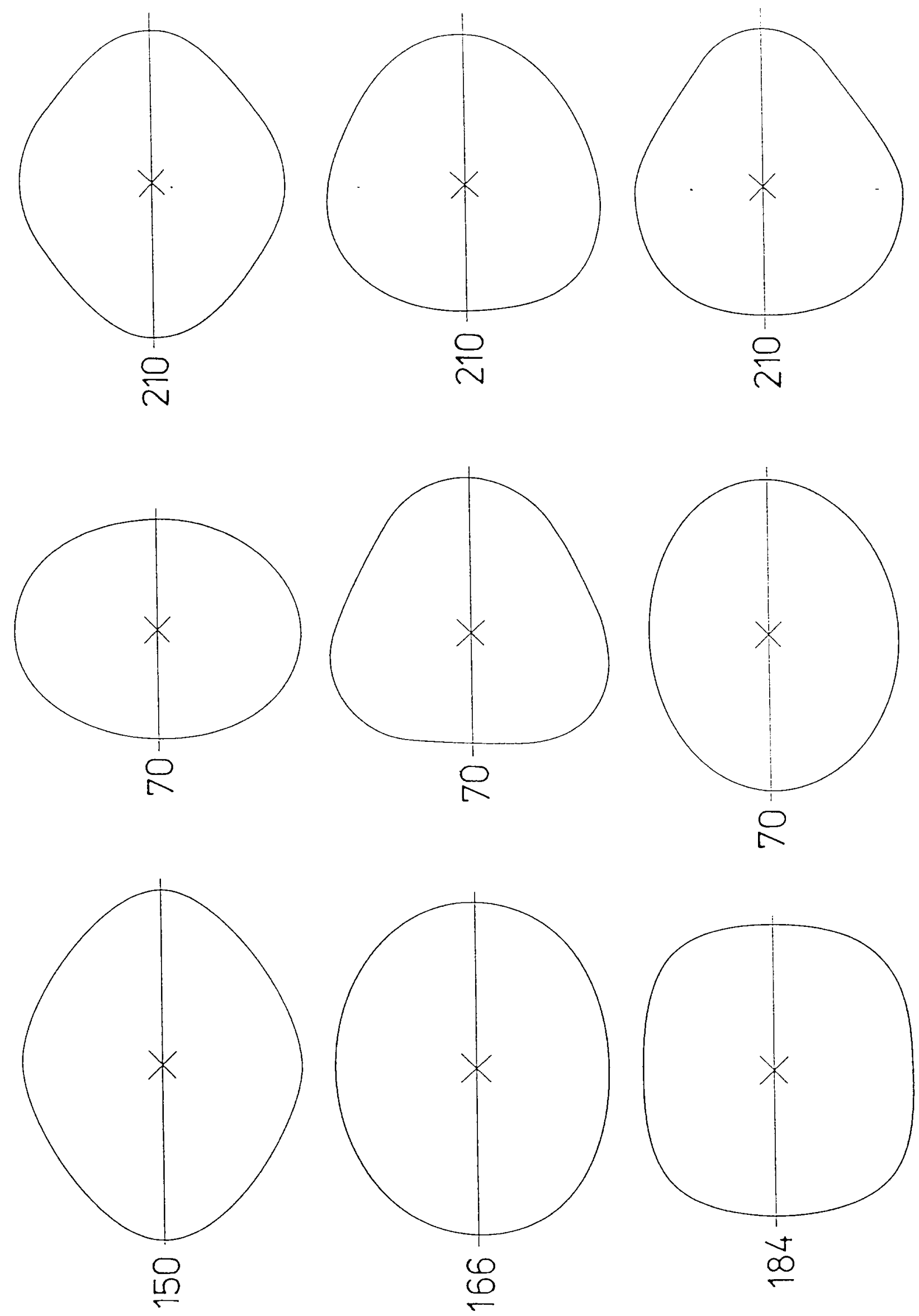

Fig. 6 


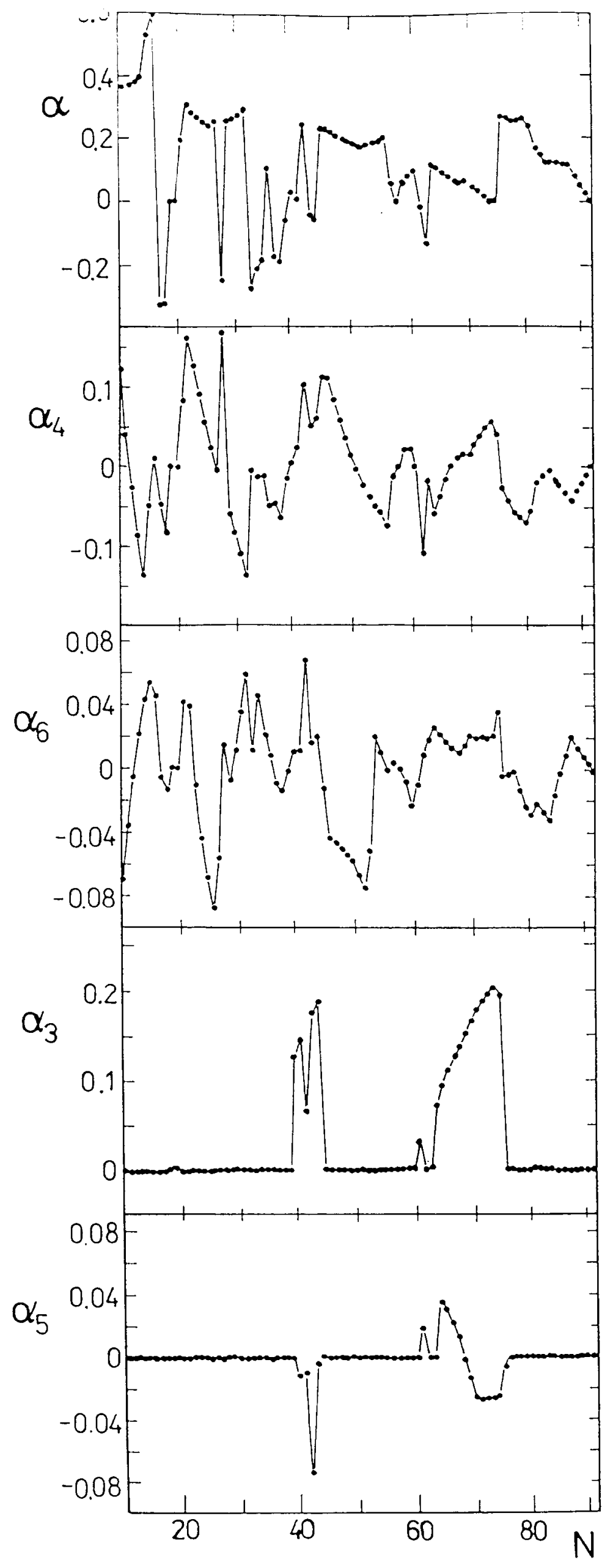

Fig. 7 


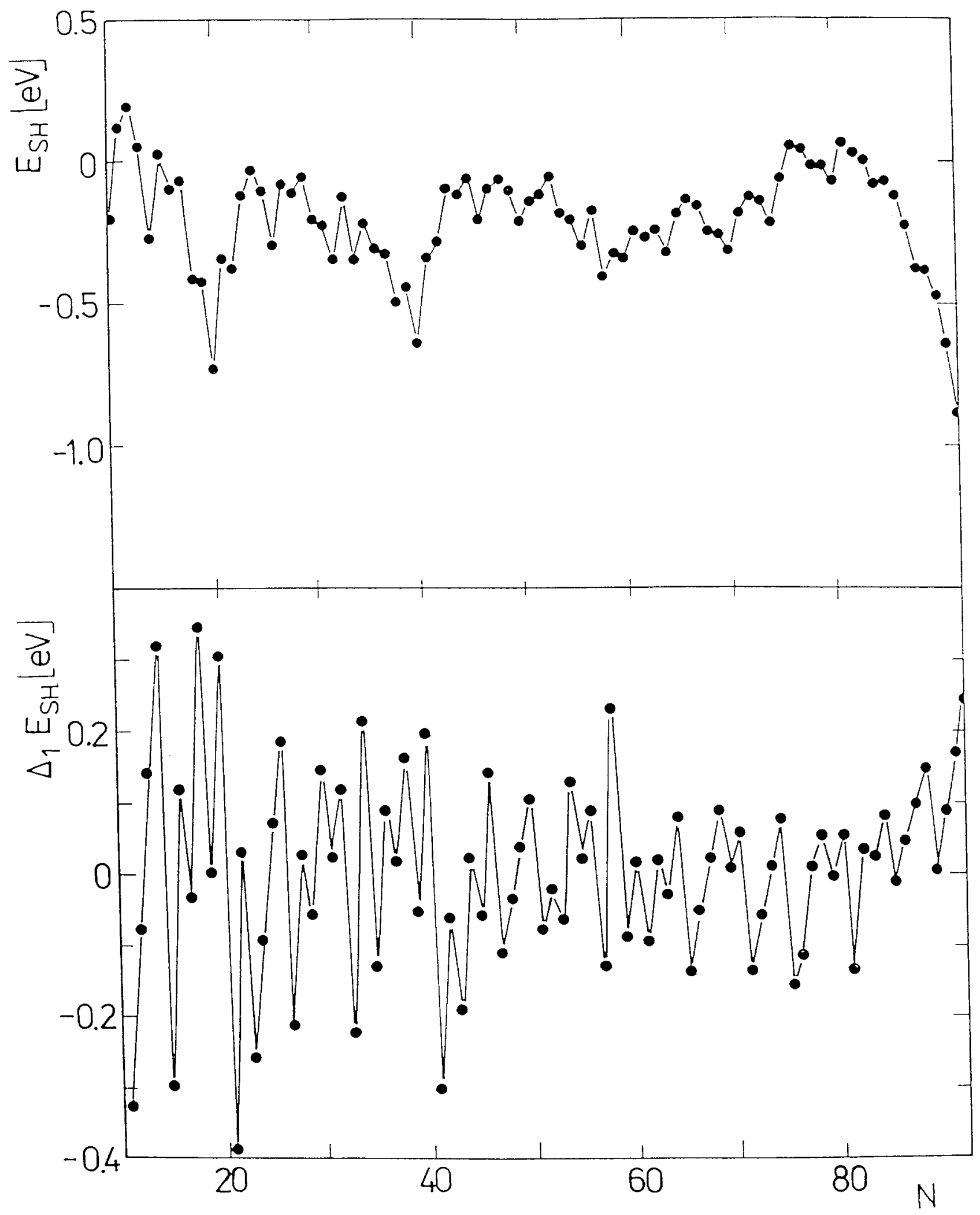

Fig. 8 


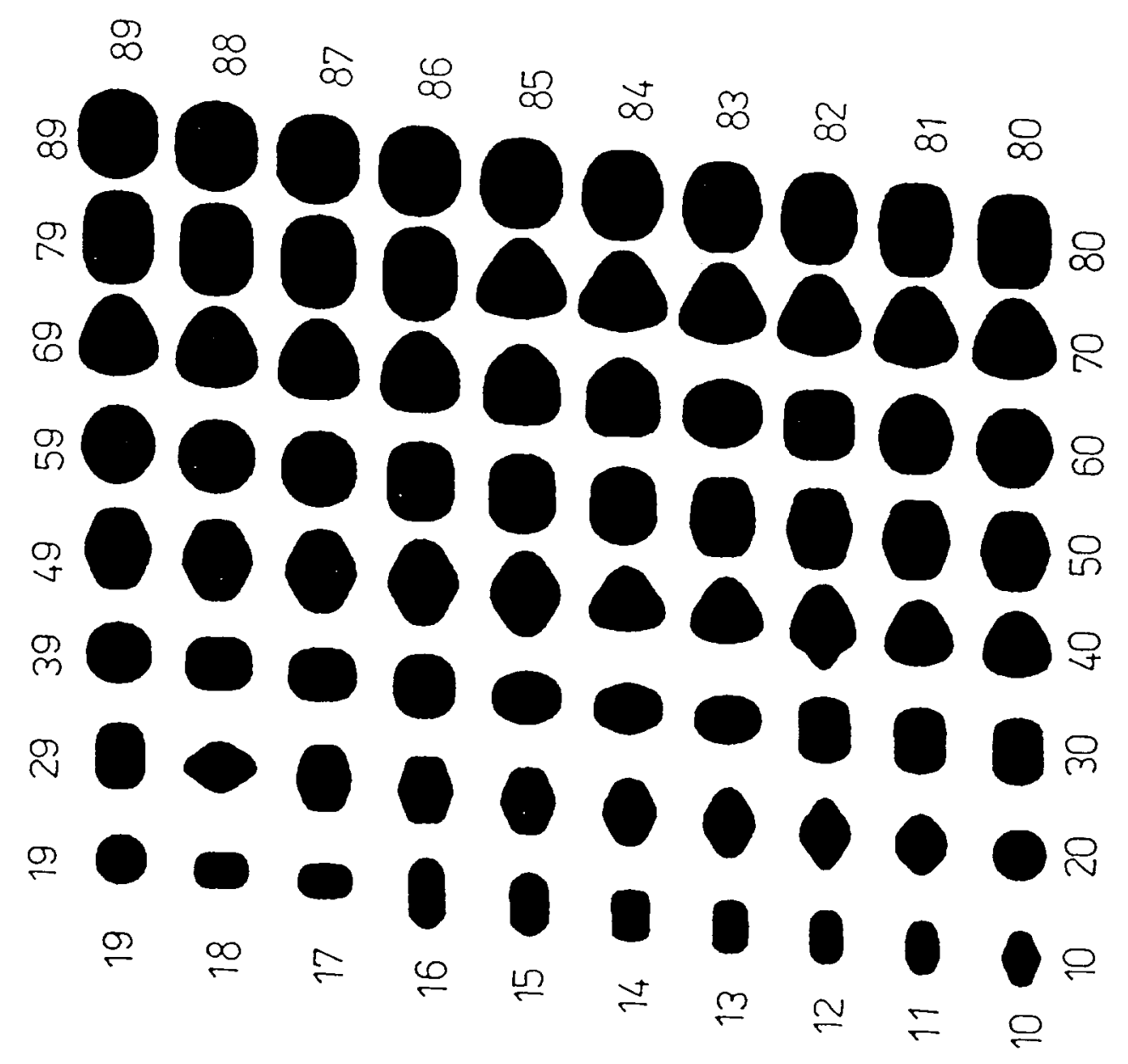

Fig. 9 


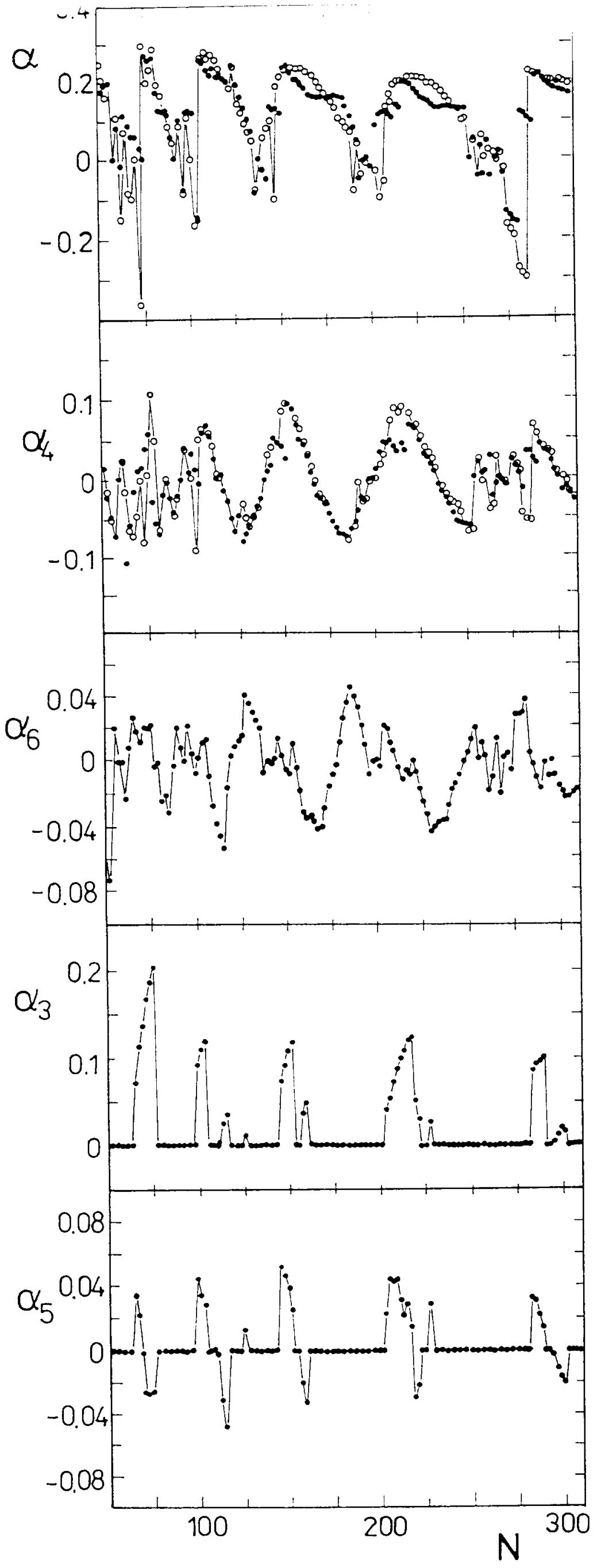

Fig. 10 


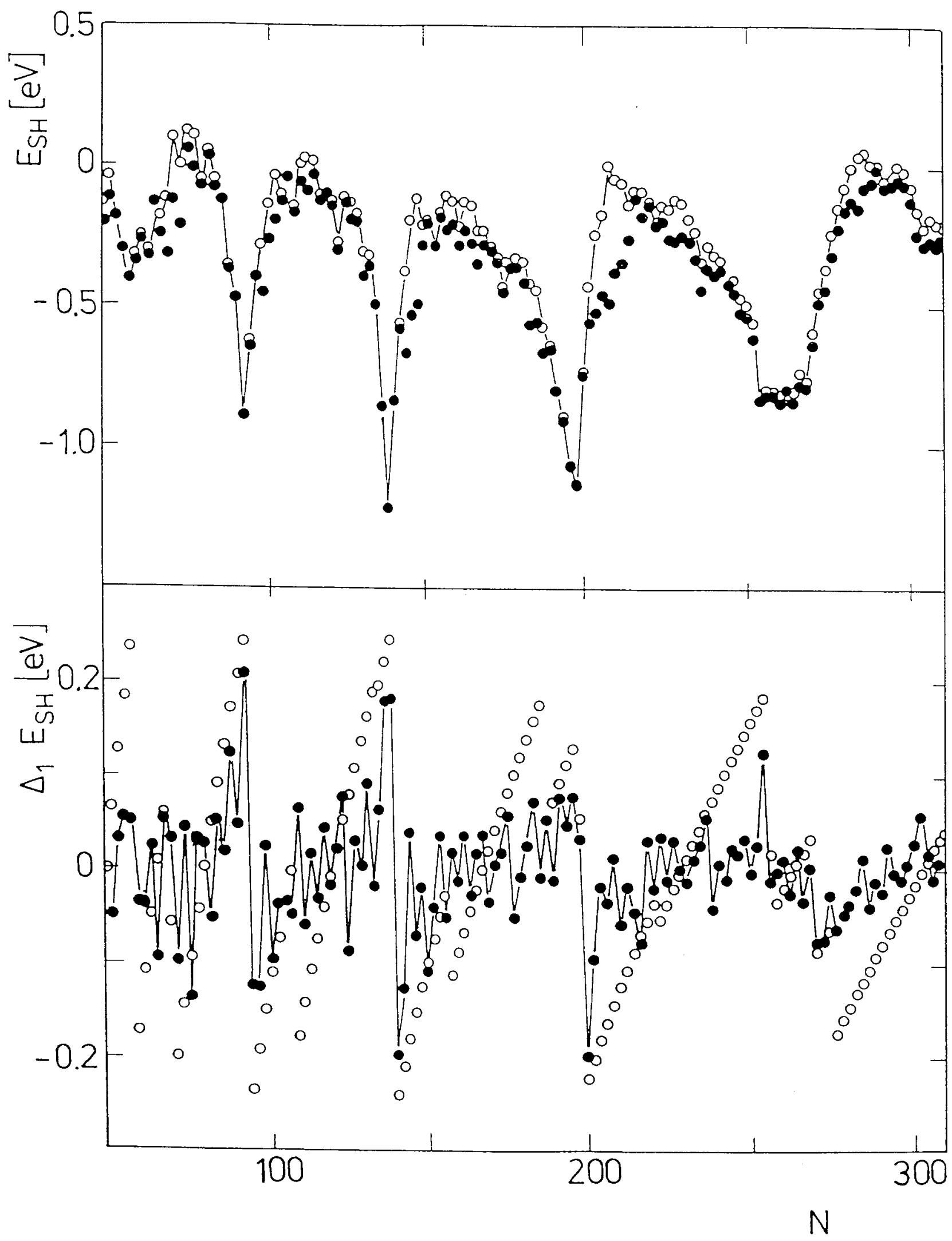

Fig. 11 


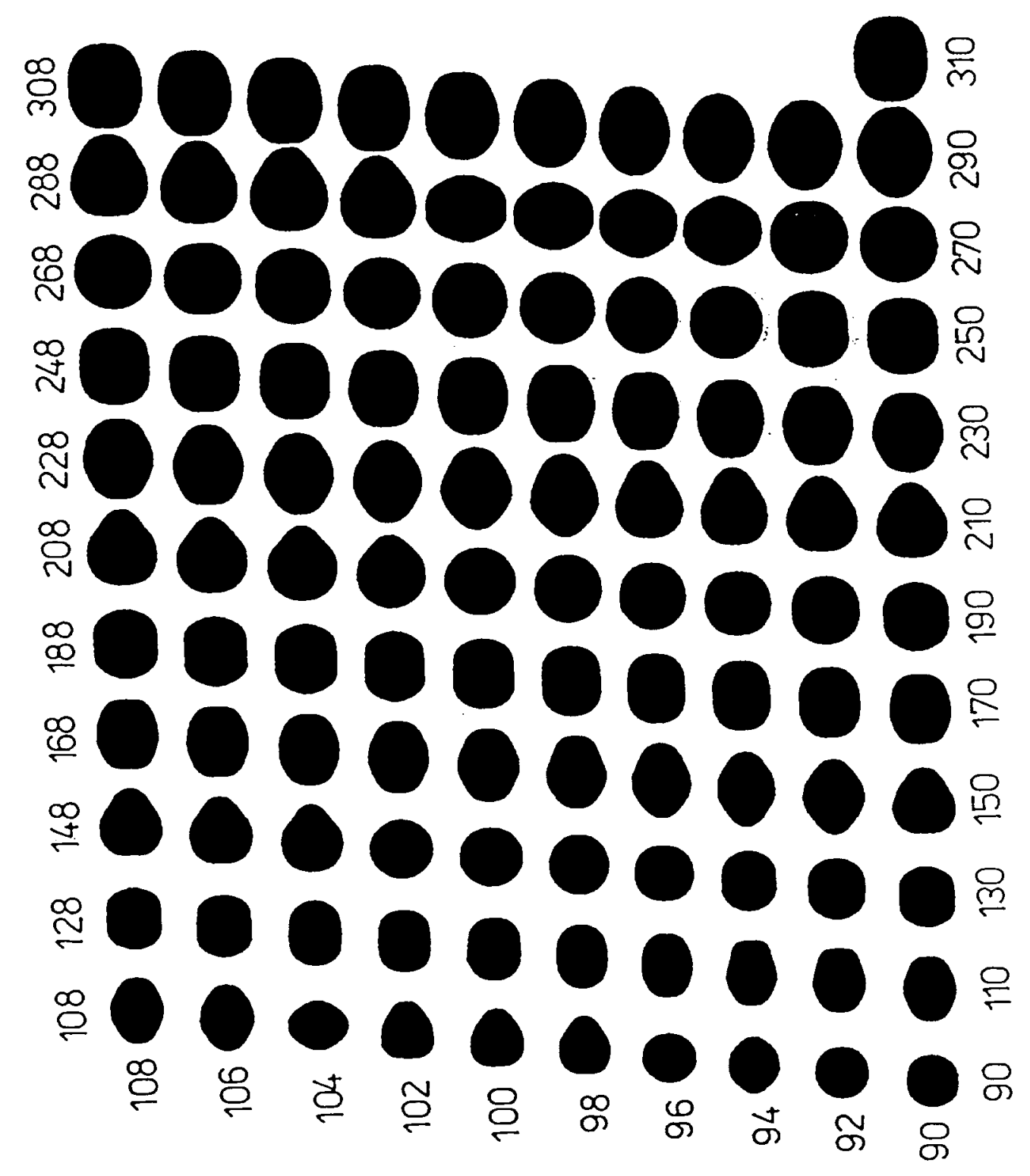

Fig. 12 


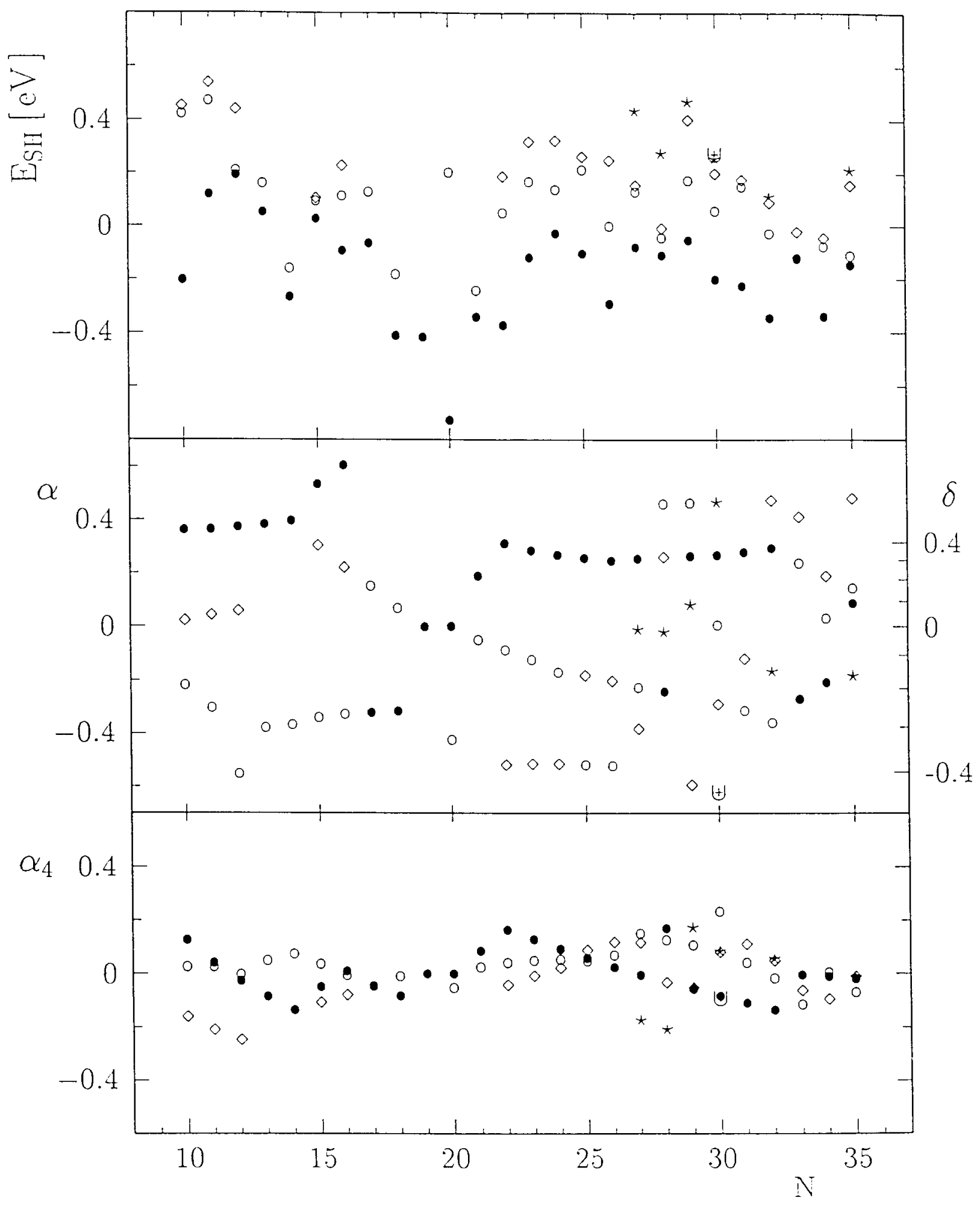

Fig. 13 


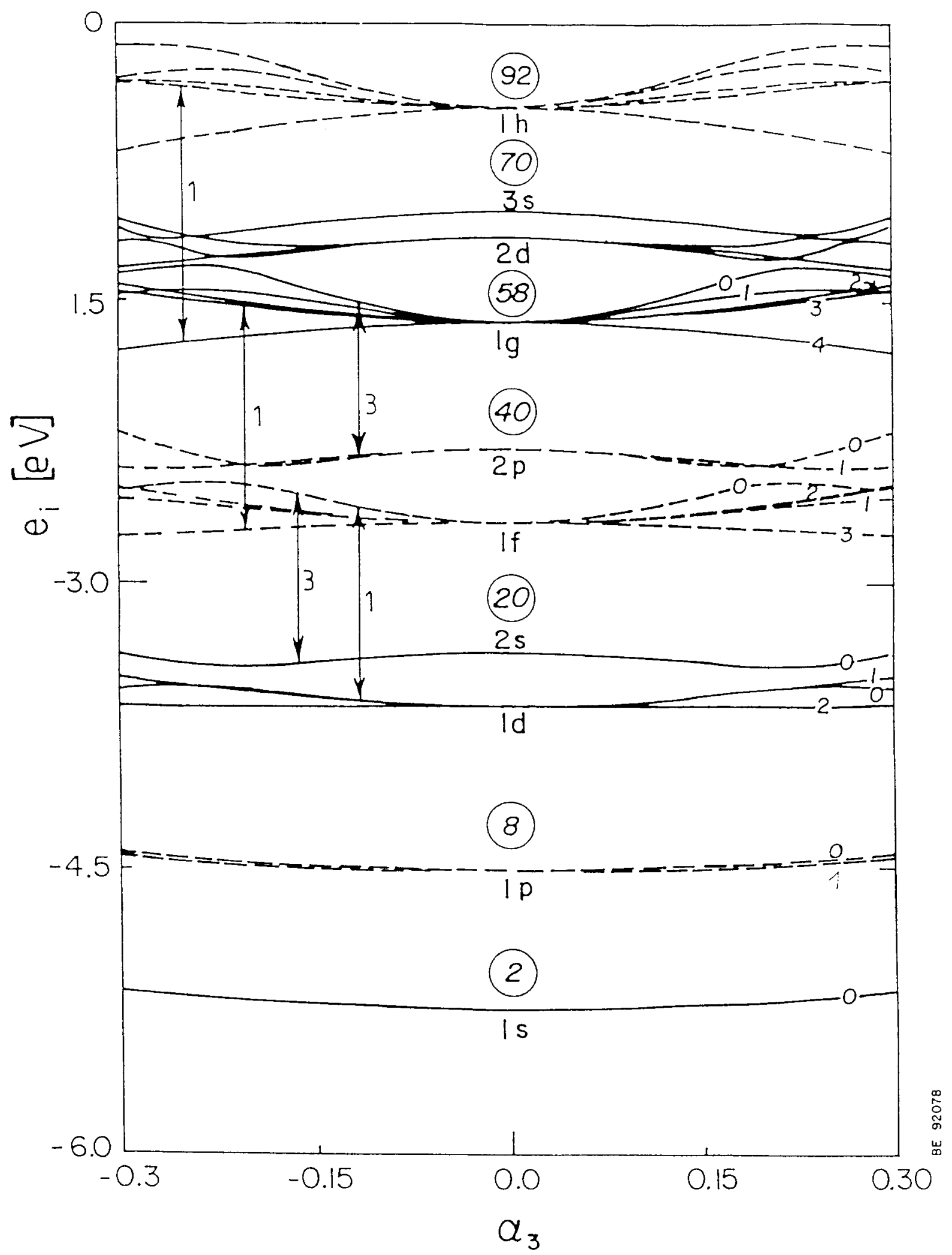

Fig. 14 


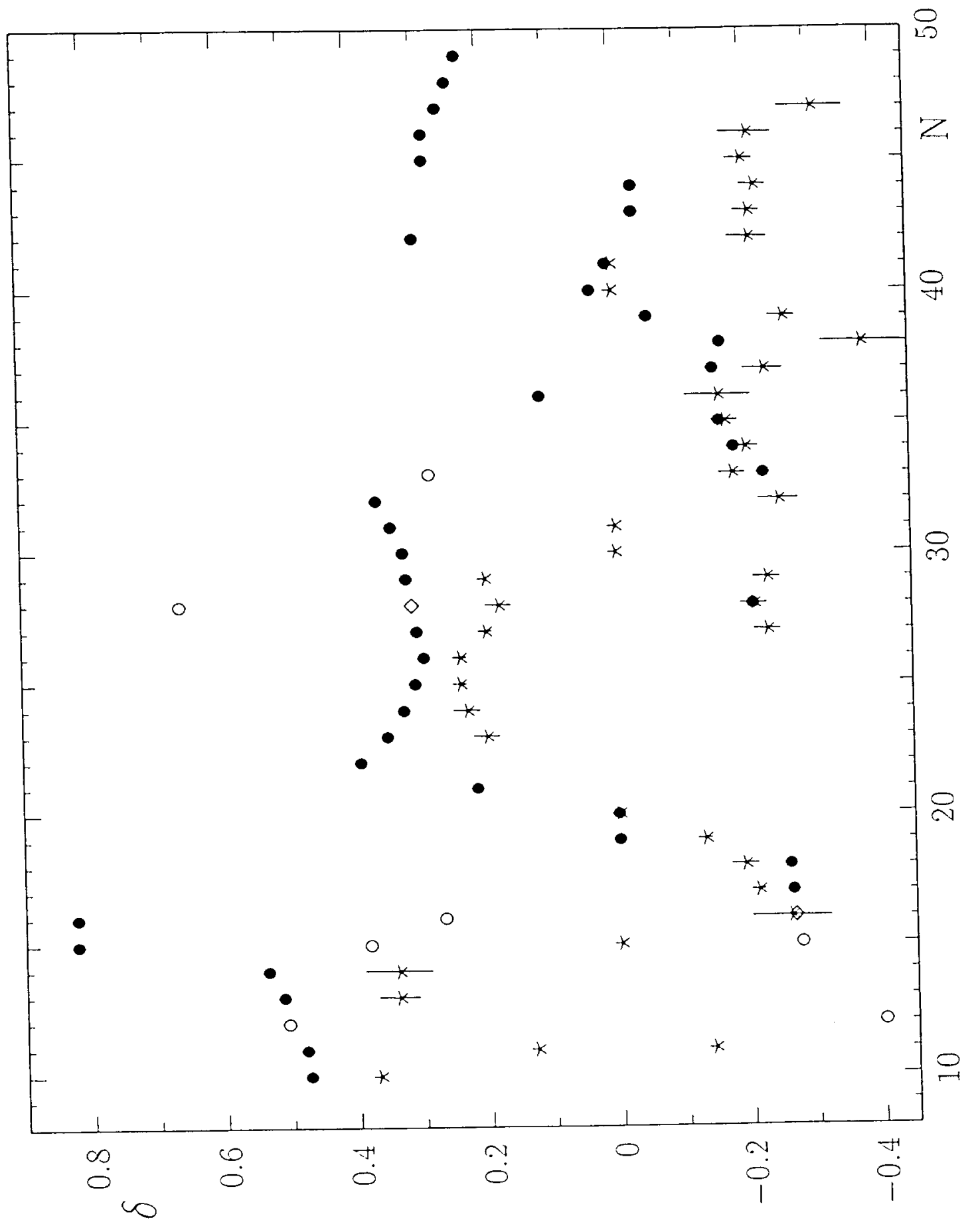

Fig. 15 


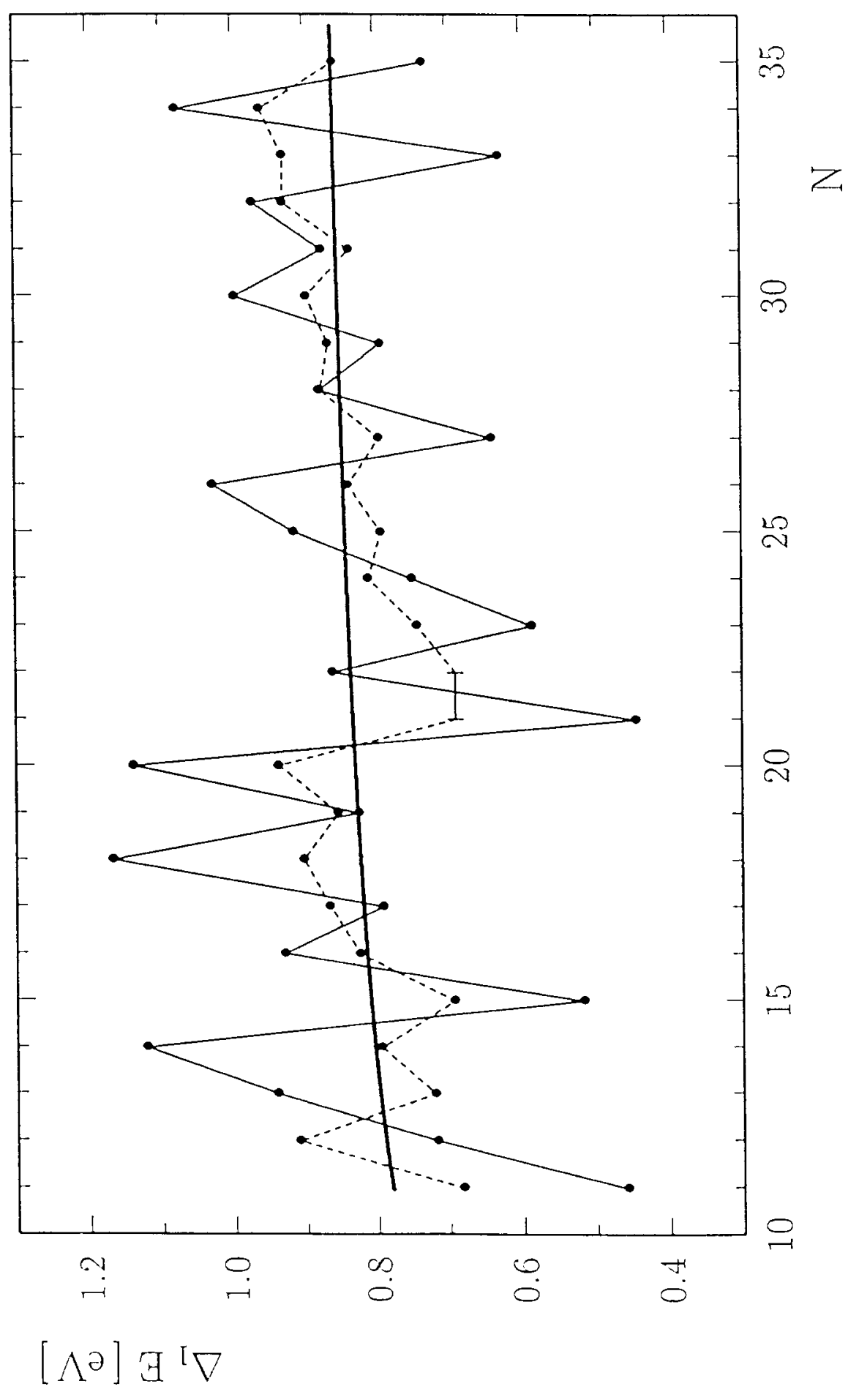

Fig. 16 